\title{
RESET
}

Recherches en sciences sociales sur Internet

4 | 2015

Les mondes du jeu

\section{Les détournements de jeux vidéo par les joueurs.}

Une incarnation du play

Detournements of videogames by players: an incarnation of play

\section{Fanny Barnabé}

\section{(2) OpenEdition}

Journals

Édition électronique

URL : http://journals.openedition.org/reset/527

DOI : $10.4000 /$ reset.527

ISSN : 2264-6221

Éditeur

Association Recherches en sciences sociales sur Internet

Référence électronique

Fanny Barnabé, "Les détournements de jeux vidéo par les joueurs. ", RESET [En ligne], 4 | 2015, mis en ligne le 21 décembre 2015, consulté le 02 mai 2019. URL : http://journals.openedition.org/ reset/527 ; DOI : 10.4000/reset.527

Ce document a été généré automatiquement le 2 mai 2019.

(c) Association Recherches en sciences sociales sur Internet 


\title{
Les détournements de jeux vidéo par les joueurs.
}

\author{
Une incarnation du play \\ Detournements of videogames by players: an incarnation of play
}

\section{Fanny Barnabé}

\section{Introduction}

\section{Approcher le play par ses traces numériques}

1 Depuis les premières heures de son histoire, le jeu vidéo engage ses utilisateurs dans une logique de détournement et de réappropriation : «la conception du premier jeu vidéo résultait déjà d'un acte ludique, qui s'est exprimé par le détournement de l'usage conventionnel d'un super calculateur du MIT. C'est en effet à partir des expérimentations informatiques d'un groupe d'étudiants se faisant appeler "hackers" que Spacewar (1962) a vu le jour " (Genvo, 2008b : 2). Cette dimension réorganisatrice de l'activité ludique est favorisée tant par la nature interactive du médium (la participation du joueur est nécessaire pour actualiser le jeu, si bien que l'œuvre vidéoludique n'existe pas en dehors de la partie), que par ses modes de production issus de la culture de masse, avec une frontière entre auteurs et publics moins étanche (la complicité des joueurs étant régulièrement et explicitement sollicitée par les développeurs ${ }^{1}$ ). Dans certains cas, cette réception active peut déboucher sur de véritables pratiques créatives permettant aux joueurs de prolonger l'expérience ludique de manière périphérique : piratage, élaboration de fictions sur les univers des jeux, réalisation de films amateur, etc.

2 Si ces pratiques existaient bel et bien avant la démocratisation d'Internet $^{2}$, ce nouveau système de communication immédiate et de partage de contenus à grande échelle a radicalement transformé les modalités de la participation des joueurs à la création. Les détournements de jeux vidéo, autrefois ponctuels et désorganisés, rassemblent aujourd'hui de larges communautés et ont développé d'importantes structures d'organisation et de régulation pour chaque activité : les praticiens se réunissent autour 
de sites qui servent à la fois d'organes de diffusion, de sélection (les œuvres y sont préalablement évaluées et validées) et de construction identitaire (les règles, les valeurs et les enjeux des pratiques y sont largement décrits et discutés). L'arrivée d'Internet a également doté les joueurs d'importants moyens de diffusion, accroissant la visibilité du phénomène, et de moyens de production pouvant avoisiner ceux des professionnels. Par ailleurs, cet outil a entraîné l'éclosion de divers dispositifs métaréflexifs sur les pratiques de détournement, à l'image des forums qui accueillent des critiques sur la qualité des productions de joueurs, des commentaires explicatifs ou encore des débats informels sur l'avenir des pratiques. L'existence de ces discours institue donc, à côté du jeu, un "plaisir du méta-jeu » (Georges, 2012). De plus, le caractère horizontal et l'immédiateté des relations interpersonnelles permises par Internet ont renforcé la confusion qui s'installait déjà entre les joueurs et les premières instances de production des jeux: «tous les acteurs du marché sont centralisés, partageant et habitant le même espace, réduisant de fait les différentes - et traditionnelles - médiations entre producteur et consommateur " (Berry, 2008b : 55). Enfin, plus généralement, Internet a été l'un des vecteurs d'une plus grande diffusion du ludique, autrement appelée "gamification $»^{3}$. Les technologies numériques dans leur ensemble entretiennent avec le jeu une relation privilégiée : elles sont, selon Vial (2012), «ludogènes ", dans le sens où elles sont particulièrement susceptibles de stimuler et de nourrir une " attitude ludique » - autrement dit, du play chez leurs utilisateurs. Cette aptitude se traduirait par l'existence d'un gameplay intrinsèque aux objets numériques, dû aux propriétés des actions qu'ils permettent (leur interactivité, leur réversibilité, leur reproductibilité instantanée, leur destructibilité, etc.). À la fois système de règles dicté par le calcul et ouverture des possibles dont le joueur peut «faire l'exercice» (Genvo, 2011: 72), le numérique semble effectivement rejoindre les critères définitionnels de la notion de gameplay tels qu'ils ont été établis par Genvo $^{4}$. Or la jouabilité du numérique n'est pas sans effet sur les pratiques de détournement qui se développent sur Internet puisque ce support, en servant de conducteur à l'attitude ludique, ouvre la possibilité d'un espace de jeu en dehors du jeu lui-même.

3 Ce dernier apport est, selon nous, central dans la compréhension des modalités selon lesquelles les réappropriations de jeux vidéo sont aujourd'hui réalisées et de l'impact qu'elles ont sur la pratique du jeu en général. En effet, l'actuelle propagation du jouable hors des seules activités identifiées comme «ludiques » et le rôle de catalyseur que semble y jouer le numérique invitent à considérer les détournements que le joueur fait subir aux jeux non pas comme des œuvres autonomes, mais comme faisant partie intégrante de son expérience ludique. Cet article aura donc pour objectif d'interroger la nature potentiellement "ludogène » des détournements de jeux vidéo par les joueurs. Dans ce but, quatre phénomènes seront ici envisagés conjointement: la rédaction de fanfictions, la réalisation de machinimas, le modding, et le speedrun ${ }^{5}$. En raison de leurs dissemblances, nous avons abordé ces domaines de manière spécifique et délimitée.

4 En effet, mobilisant la perspective des "sciences du document ", ces quatre pratiques ont été étudiées non sous l'angle d'une observation directe, mais à travers les "traces d'usage » (Davallon, Noel-Cadet et Brochu, 2003) observables dans les œuvres produites et dans leurs paratextes. Nous nous situons, en somme, dans la ligne directe de l'analyse de discours, selon laquelle il est possible de discerner, dans tout texte, des traces de son contexte d'énonciation. La notion de trace - qui permet d'identifier « des figures de la pratique et des représentations d'identité sociale» - est ici à entendre comme «une 
textualisation des pratiques sociales dans un dispositif sémiotique qui forme un ensemble signifiant» (Flon et al., 2009: 2, version numérique). Pour envisager l'ensemble de ce "dispositif sémiotique» et donc éviter d'isoler les œuvres de la situation de communication dans laquelle elles s'inscrivent, le corpus étudié ne s'est pas borné au contenu et à la forme des œuvres produites : il s'est également composé des discours de praticiens qui s'articulent autour d'elles et en constituent le paratexte. Ces discours n'ont pas été provoqués, mais abordés, eux aussi, à travers leurs traces déjà présentes sur Internet: présentations des détournements faites par leurs auteurs, commentaires des récepteurs, discussions sur la pratique consignées dans les forums, définitions de l'activité élaborées dans les foires aux questions des sites web, interviews des producteurs (dans les cas précis des machinimas) et présentations de soi fournies par les participants sur leurs pages de profil (dans le cas des fanfictions). L'objectif était ainsi de ne pas réduire ces créations à des productions statiques, mais de les envisager comme des processus de communication dynamiques dans lesquels l'organisation de la communauté participe à l'élaboration du sens.

5 Si ce matériau se caractérise par une hétérogénéité certaine, la visée de la présente étude est de mettre au jour l'existence de lignes de force communes aux quatre pratiques et d'en élaborer un modèle explicatif global; en outre, "paradoxalement, analyser les usages là où on les attend le moins - c'est-à-dire dans les objets médiatiques - permet de rompre avec une approche uniquement sémiotique du texte, uniquement technique du site et uniquement sociale de l'usage " (Davallon, Noel-Cadet et Brochu, 2003:57). Il importera toutefois de souligner le caractère exploratoire dudit modèle et la nécessité de le compléter par d'autres enquêtes, dont cet article pourra constituer les premières bases.

\section{Quels détournements?}

6 La première forme de détournement étudiée, la fanfiction, se définit comme l'écriture par les «fans » de récits inspirés d'univers fictionnels préexistants. Les amateurs prennent la plume pour combler une faille du scénario original, développer un aspect qui n'était qu'esquissé, parodier un passage canonique, etc. La pratique prend origine dans les années 1960-1970 au sein des communautés de fans de la série Star Trek qui s'échangeaient leurs textes sous forme de fanzines (voir Jenkins 1992 et 2006). Elle n'est donc pas propre au médium vidéoludique mais n'en demeure pas moins un phénomène d'envergure : le 23 mars 2014, on trouvait par exemple sur Fanfiction.net (site de référence en raison de son ancienneté, de son orientation "généraliste » et du grand nombre de fictions qu'il héberge ${ }^{6}$ ), pas moins de 70900 fanfictions associées au seul univers du jeu Kingdom Hearts, 70100 textes inspirés de celui de Pokémon, 38800 basés sur Final Fantasy VII, etc, toutes langues confondues. Dans le cadre de ce travail, le domaine a été étudié à travers un corpus de trente textes inspirés, précisément, des jeux Kingdom Hearts (ce sont ceux qui ont donné lieu au plus grand nombre de réécritures) et recueillis sur Fanfiction.net. Deux critères ont présidé à leur sélection : la langue (celle-ci étant au centre de la pratique, nous avons jugé pertinent de nous limiter aux sources en français) et la popularité (il s'agit des trente textes les plus fréquemment enregistrés comme « favoris » par les lecteurs).

7 Les machinimas ${ }^{7}$, ensuite, sont des films d'animation réalisés à partir des moteurs graphiques des jeux. Si, au départ, les vidéos ainsi produites étaient principalement utilisées pour faire part à la communauté d'une impressionnante performance ludique ${ }^{8}$, 
la pratique s'est rapidement diversifiée (dès la fin des années 1990 selon Georges et Auray, 2012a) et a donné naissance à plusieurs genres: machinimas humoristiques ou parodiques, clips musicaux, spectacles d'improvisation, longs métrages de fiction, etc. Contrairement à celui des fanfictions, le domaine du machinima ne dispose pas d'une base de données centralisée qui embrasserait tous les jeux et tous les types de productions : les créateurs de machinimas (ou "machinimakers ») diffusent leurs vidéos via des réseaux qui leur sont propres ou se réunissent sur des sites consacrés au jeu qu'ils utilisent. Pour délimiter un corpus cohérent, nous avons donc décidé de nous focaliser sur les machinimas réalisés à partir de la série de jeux $\mathrm{Halo}^{9}$. Le fait qu'il s'agisse d'une licence particulièrement exploitée par les machinimakers (à côté de jeux tels que World of Warcraft, Les Sims, City of Heroes,...) et qu'elle soit fréquemment citée dans les travaux académiques traitant du sujet ${ }^{10}$ a motivé ce choix. Mais celui-ci s'est trouvé conforté au regard de la notoriété acquise, dans les années 2000, par certaines des vidéos issues de ces jeux , vidéos qui ont participé à faire connaître le machinima à un public élargi et qui ont influencé, en retour, le développement de la série Halo, à l'image des séries Red vs. Blue et This Spartan Life $e^{11}$.

8 Le modding, en troisième lieu, désigne la modification de certains éléments d'un jeu (personnages, niveaux, apparences des objets...) dans le but d'en créer une version améliorée ou, à l'occasion, de produire une nouvelle œuvre à part entière, la possibilité de manipuler le code d'un jeu vidéo pouvant être préméditée par les concepteurs ou se développer à leur insu. Comme pour le machinima, la pratique du modding ne dispose pas d'un site généraliste qui centraliserait l'ensemble des productions (les «moddeurs » se rassemblant plutôt en sous-communautés autour des jeux qu'ils altèrent). Malgré tout, il existe au sein de ces différents groupes une base de données qui se distingue par son ampleur (elle compte actuellement 7038480 membres) et sa relative ouverture (elle rassemble des mods portant sur nonante-deux jeux différents) : il s'agit de Nexus mods and community. L'œuvre qui a suscité le plus de réécritures sur ce site étant The Elder Scrolls V: Skyrim $^{12}$, nous nous sommes concentrée sur les mods issus de ce jeu et avons limité l'analyse aux cinquante premiers du classement par ordre de popularité (c'est-à-dire à ceux qui ont reçu le plus de votes).

Enfin, le speedrun consiste à tenter de terminer un jeu le plus vite possible (notamment en exploitant ses failles techniques ou "glitchs») et à réaliser une vidéo de cette performance. Dans le but de parfaire leur record et de parvenir à un temps théoriquement idéal, il arrive que les joueurs utilisent les outils fournis par les émulateurs pour ralentir le jeu et enregistrer les différentes commandes actionnées : on parle alors de " tool-assisted speedrun» (ou «TAS»). Speedrun et TAS disposent chacun d'un site de référence, à savoir Speed Demos Archive et TASVideos respectivement ${ }^{13}$. Le corpus étudié s'est donc fondé sur les productions diffusées sur ces deux hébergeurs: il se compose des vingt-cinq premières vidéos de TASVideos ayant obtenu une " étoile » (grade octroyé aux "runs" les plus populaires) et des trente-six premières de Speed Demos Archive classées par ordre de parution des jeux, ce deuxième site ne disposant pas de marqueurs mesurant la réputation des productions.

Comme le révèlent déjà ces brèves présentations, la forme sous laquelle se présentent les détournements peut s'éloigner fortement du jeu vidéo d'origine: les fanfictions sont des textes, le speedrun et les machinimas des vidéos, et les mods eux-mêmes ne sont pas toujours à l'origine d'un renouveau du gameplay mais peuvent venir corriger une composante du jeu qui avait été mal programmée (tel le piètre aspect de l'eau, selon les 
joueurs, dans The Elder Scrolls V: Skyrim). Le passage d'un système sémiotique à l'autre entraîne nécessairement des pertes au niveau de la jouabilité: l'interactivité, la répétabilité et le gameplay propres au jeu vidéo sont, en apparence, absents de certains détournements - du moins, ces propriétés n'y apparaissent pas sous leur forme originale de «marqueurs pragmatiques de jouabilité " permettant la transmission d'une « signification partagée de jeu» (Genvo, $2011: 76$ et 69). Ainsi, certains mods ne sont pas jouables et les textes de fanfictions, tout comme les vidéos que sont les machinimas et les speedruns, ne se présentent pas explicitement comme des dispositifs interactifs, pourvus de règles et dotés d'une "replay value ». Toutefois, la nature ludique de l'œuvre originale ne semble pas être sans effet sur les créations qui s'en inspirent, mais plutôt en conditionner la réalisation : c'est, en tout cas, la thèse que cet article tâchera de défendre en s'inscrivant dans le paradigme théorique des play studies.

\section{Le jeu comme expérience}

11 Dans son ouvrage Philosophie des jeux vidéo, Triclot (2011) pointe la tendance générale des game studies à mettre à distance le jeu vidéo et à le définir comme un système formel stable, sans tenir compte de l'influence qu'exerce sur lui la présence du joueur. Cette approche facilite certes l'appréhension théorique du jeu mais évacue la question des différentes expériences qui peuvent être faites d'une même œuvre. En réaction à cette construction idéale et figée, Triclot propose de combler le vide laissé par les game studies en créant des play studies, qui s'interrogeraient sur l'expérience ludique et non plus seulement sur le jeu en tant qu'objet. Ce changement de paradigme comporte l'immense avantage de ne pas nier la variabilité intrinsèque des œuvres vidéoludiques et de ne pas exclure de la réflexion les usages qui débordent du jeu en tant que structure. Selon cette perspective, il est possible d'établir une continuité entre la pratique d'un jeu vidéo et la création ou consommation de ses détournements, puisque tous ces usages font partie d'une même expérience ludique : l'attitude libre du joueur (le play) ne se limite pas au dispositif qui l'a fait naître (le game, le jeu et ses règles) mais le dépasse et le prolonge hors de son espace habituel ; ce processus rejoint, selon nous, la notion de transformative play, définie par Salen et Zimmerman (2004: 305) comme la possibilité, pour le joueur, d'altérer la structure même d'un jeu (le game) par sa manière - imprévue et créative - d'y jouer.

12 L'optique des play studies implique néanmoins un important problème méthodologique : la difficulté d'objectiver les phénomènes étudiés. Or, si l'on considère les détournements de jeux vidéo comme le résultat d'un transfert de l'activité du joueur sur différents supports, ces mêmes réappropriations pourraient alors constituer, pour le chercheur, un moyen concret d'appréhender le play des joueurs. Les détournements peuvent, en effet, apparaitre comme autant de strates donnant corps et visibilité à des expériences individuelles du jeu (qui, sans ces « actualisations ", ne serait qu'un paradigme doté d'une existence "virtuelle $»^{14}$ ), celles-ci venant s'incarner dans des objets montrables. La variabilité de la partie peut ainsi être temporairement figée et donc transmise, puisqu'il devient possible de revivre, bien que sous d'autres conditions, l'expérience particulière d'un autre joueur: "[...] the multiplicity of choices potentially generated through interaction within a virtual environment become contained as a singular and repeatable trajectory of actual choices made by the producer [...] » indique, au sujet des machiminas, le chercheur Gareth Schott (2011: 116). 

(et notamment au play), il importe également de mentionner qu'en figeant cette expérience, ils peuvent générer un autre dispositif ludique, c'est-à-dire un nouveau game pourvu de règles, d'objectifs, de valeurs et de conventions. Par exemple, si la rédaction d'une fanfiction représente un acte individuel d'interprétation conditionné par la lecture que le joueur-auteur a fait du jeu dont il s'inspire, celle-ci peut, en circulant, être réinvestie par la communauté jusqu'à devenir un modèle collectif, une nouvelle contrainte pour les réappropriations futures ${ }^{15}$. Néanmoins, le nouveau game produit par cette forme d'institutionnalisation est lui-même loin de constituer une œuvre figée : les détournements ne sont pas des objets finis mais des processus dynamiques, en attente continuelle d'être enrichis, commentés ou eux-mêmes modifiés. Pour preuve, le célèbre mod du FPS ${ }^{16}$ Half-Life nommé Counter-Strike a lui-même été altéré de nombreuses fois par ses utilisateurs (qui en remanient l'aspect, le gameplay, etc.). Les réécritures de jeux vidéo semblent donc elles-mêmes appeler une participation. En ce sens, elles ne paraissent pas totalement s'émanciper de la logique du game design.

Nous tenterons, dans les pages qui suivent, de décrire les différents aspects (formels et communautaires) des détournements qui établissent une continuité entre ces pratiques et celle du jeu. Parmi les traits d'union qui sont ressortis de nos corpus, trois seront ici développés : la parenté du jeu et de la réécriture en tant que performances, leur tendance commune à l'interactivité et l'identité de posture entre le joueur et le créateur. Ces angles d'approche permettront de concevoir fanfictions, machinimas, speedruns et mods non pas comme de simples textes, vidéos ou améliorations techniques, mais comme des objets sémiotiques hybrides, mêlant les codes de la littérature, du cinéma, de la performance ou même du travail à ceux du jeu.

\section{Le jeu et le détournement : deux formes de performance}

15 Le concept de « performance $\|^{17}$ constitue un premier point de passage entre le jeu et les usages qui le réinvestissent. En effet, les détournements prolongent l'activité du joueur en se présentant eux aussi comme des performances qui peuvent être compétitives (la performance comme record) ou « théâtrales » (la performance comme interprétation).

\section{La performance comme record}

16 Bien que les visées de " performance » et de " compétition » ne soient pas au cœur de la définition des quatre phénomènes étudiés et ne ressortent pas particulièrement des discours des praticiens, l'observation des modes de production des détournements permet toutefois d'avancer que ces pratiques entraînent une forme de progression dans la maîtrise du jeu original ${ }^{18}$. En effet, la vidéo de speedrun et le record qu'elle établit attestent du développement préalable, chez le joueur, d'une importante compétence dans plusieurs aspects du jeu (la vitesse, le maniement précis de l'avatar), tandis que l'éventuelle présence de bugs exploités témoigne d'un travail antérieur de recherche. De même, le travestissement des mécaniques ludiques dans les machinimas (le fait d'utiliser la mort d'un avatar dans Halo pour le faire s'allonger et simuler son sommeil, par exemple) laisse supposer que le machinimaker a pris connaissance de ces mécaniques et est capable de les exécuter. La publication de mods fonctionnels invite elle aussi à inférer, 
chez les producteurs, une compréhension avancée du jeu modifié et même, dans certains cas, de compétences en programmation ${ }^{19}$. Enfin, l'observation des fanfictions les plus valorisées par la communauté et de la manière dont celles-ci réagencent les éléments narratifs de l'œuvre originale (personnages, lieux, situations,...) révèle généralement chez les fans une connaissance détaillée de l'univers investi, voire des fanons qui s'en inspirent ${ }^{20}$; selon Kaplan, « a large part of the fannish experience lies in analyzing the source texts of fandom » (Kaplan, 2006 : 135).

Ce souci de maîtrise du texte-source transparaît, entre autres, dans les débats que suscitent ses interprétations plus «libres ». C'est notamment le cas de l'« OOC » (out of character), procédé qui consiste, au sein d'une fanfiction, à modifier un personnage sans respecter la manière dont il a été décrit dans l'œuvre originale, comme faire d'un héros timide un grand séducteur, par exemple. Si de telles libertés sont régulièrement prises par certains fans, elles suscitent de nombreuses réserves de la part des auteurs euxmêmes et peuvent être un motif de critique $^{21}$. Les modes de production des détournements permettent, en somme, de postuler chez leurs auteurs la préexistence d'une pratique approfondie de certains aspects du jeu (qu'il s'agisse du système, de la narration, de la course, ou de la manipulation des avatars).

Il faut toutefois de préciser que cette première manifestation de la performance dans les détournements d'œuvres ludiques ne va pas jusqu'à définir, pour l'activité, un praticienmodèle qui serait un "joueur d'élite »: si l'œuvre dérivée porte - de manière plus ou moins marquée - la trace d'une certaine maitrise du jeu, cette maîtrise ne constitue pas un pré-requis à l'entrée dans la pratique. Autrement dit, loin de poser la performance comme une norme réglementant la participation, les praticiens la définissent plutôt comme un modèle auquel l'exercice du détournement doit petit à petit faire accéder. En ce sens, l'apprentissage n'est pas envisagé comme une activité périphérique, isolée de la création, mais comme « une façon de participer à des pratiques sociales, un statut, un mode d'appartenance à une communauté, une façon "d'en être" ", rappelant ainsi la notion de « communauté de pratique » (Berry, 2008a : 13).

Cette valeur fédératrice attribuée à la progression des praticiens n'empêche cependant pas de s'interroger sur la provenance de cet idéal de performance vers lequel elle tend: l'une des réponses qui pourrait être proposée tient dans l'origine ludique des quatre activités, puisque l'on y retrouve une même valorisation de la compétence, du niveau de jeu (ou «skill»), que celle qui peut être observée dans les usages performatifs du jeu vidéo. En dehors des œuvres, l'existence latente d'une performance "compétitive » se marque, entre autres, dans l'omniprésence des systèmes de classement, visible au sein des quatre pratiques : la compétence des joueurs-créateurs ne cesse, en effet, d'y être évaluée selon divers critères. La reconnaissance d'une fanfiction se marque au nombre de "reviews $»^{22}$ qu'elle a reçues, nombre mentionné dans la description du texte sur Fanfiction.net et pouvant servir de « filtre » pour orienter la recherche dans la base de données ; celle d'un machinima s'observe au nombre de fois qu'il a été visionné, sans quoi il risque de passer inaperçu dans les jungles que sont YouTube, Dailymotion, etc. ; un mod s'évalue au nombre de téléchargements ou de votes positifs qu'il a reçus, ceux-ci pouvant lui donner accès à une " top list ", comme sur le site Nexus; et un speedrun ne sera validé que s'il réalise un nouveau record, les performances dépassées étant vouées à disparaitre ou à recevoir la mention «obsoleted» sur le site observé. Ces récompenses quantifiables, qui ne sont pas sans rappeler le fonctionnement de nombreux jeux vidéo, peuvent également servir à mesurer un progrès ou l'ampleur de l'effort fourni : une fanfiction qui aura été pré-évaluée 
par plusieurs bêta-lecteurs ${ }^{23}$ gagnera en légitimité puisque la maitrise de la langue - si elle n'est pas une condition pour publier - reste tout de même un aspect valorisé par la communauté24 ; de même, un tool-assisted speedrunner ne manquera pas de mentionner, dans la description de sa vidéo, le nombre de sauvegardes (re-record) effectuées pour atteindre son record, car c'est une indication du temps passé à la réaliser ${ }^{25}$.

Les différents groupes observés manifestent, en outre, un réel souci didactique visant à faciliter l'entrée dans leurs pratiques. En effet, les sites étudiés regorgent de ressources pédagogiques: conseils d'écriture et propositions de relectures sur les forums de Fanfiction.net ${ }^{26}$, constitution d'une encyclopédie participative (un « wiki ») sur le site Nexus comprenant des tutoriels de tous ordres et des informations sur les jeux fréquemment " moddés ${ }^{27}$, partage de techniques et de choix de parcours chez les speedrunners et les tool-assisted speedrunners ${ }^{28}$, ou encore guides vidéo recensant conseils et astuces pour la création de machinimas ${ }^{29}$. Encore une fois, l'apprentissage nécessaire à ces différents types de détournement, loin d'être dissimulé, est intégré aux valeurs régissant la performance créatrice, tout comme les jeux vidéo intègrent généralement, dans leur dispositif, une courbe croissante de difficulté.

\section{La performance comme interprétation}

21 Si le jeu vidéo et ses détournements ont en commun leur exigence compétitive, la performance qui s'y joue peut également être envisagée comme une interprétation. Le jeu vidéo se rapproche, en effet, des arts allographiques tels que définis par Goodman (2005). Ceux-ci se différencient des arts autographiques (comme la peinture, où le support matériel de l'œuvre fait sens et où l'original est distingué de ses reproductions), dans le sens où ils se présentent comme des "partitions " permettant d'exécuter une même œuvre une infinité de fois. Or les quatre formes de détournements envisagées semblent conserver cette nature de script (au sens théâtral du terme) qu'il conviendrait de " performer ».

C'est le cas des fanfictions qui, selon Coppa (2006: 226), entretiennent davantage de similitudes avec le théâtre qu'avec la littérature. Envisager ces textes à l'aune de critères dramatiques permet en effet à cette chercheuse d'éclairer trois de leurs principales caractéristiques stylistiques. Premièrement, l'attention portée au corps des personnages plutôt qu'aux intrigues et aux idées: dans ces textes, le scénario n'est, bien souvent, qu'un prétexte à la mise en scène des corps dans d'autres contextes que ceux dont ils sont issus. Autrement dit, plutôt que d'élaborer un récit, les fanfictions cherchent avant tout à réutiliser des personnages préexistants et à les faire performer de nouvelles «lignes de comportement » (behavioral strips, toujours au sens théâtral ; voir Coppa, 2006 : 230). En témoigne l'un des partis-pris organisationnels du site Fanfiction.net, qui consiste à utiliser les protagonistes comme des outils de classification: les noms des personnages convoqués dans une fiction sont effectivement mentionnés dans sa description (au même titre que le genre dans lequel elle s'inscrit) et peuvent servir de filtres facilitant la recherche du lecteur. Deuxièmement, la conception de la fanfiction comme un drame permet d'envisager l'importance qui est accordée aux répétitions non plus comme un échec du point de vue des conventions littéraires mais comme une nécessité dans l'économie générale de cette esthétique. La valorisation de la répétition rejoint, dans le domaine vidéoludique, l'attention que les joueurs portent à la « replay value » d'un jeu, c'est-à-dire à la possibilité d'y rejouer plusieurs fois sans que ce jeu perde de son intérêt. 
Le texte de fanfiction, d'autre part, n'est pas considéré comme un objet sacré, intouchable, mais comme un réservoir de situations pouvant, à leur tour, être réutilisées : la fiction dans son ensemble est soumise à une logique allographique. Enfin, ce changement de paradigme permet à Coppa de souligner que la lecture de fanfictions est loin d'être une activité solitaire : «fan fiction is not merely a text, it's an event. [...] there's a kind of simultaneity to the reception of fan fiction, a story everyone is reading, more or less at the same time, more or less together " (2006:239). La nature événementielle de cette pratique d'écriture se marque, entre autres, dans la fréquente mise en place de concours, de défis lancés par les lecteurs ou les auteurs ${ }^{30}$ et de célébrations ${ }^{31}$. Comme le théâtre qui n'existe pleinement que lorsqu'il est performé face à un public, la fanfiction s'appuie donc aussi sur le sien pour pouvoir exister.

La dimension centrale de la performance comme interprétation est encore plus perceptible dans le cas des machinimas puisque le joueur s'y change explicitement en metteur en scène, voire en acteur : il doit manipuler les avatars pour leur faire effectuer des actions évocatrices et peut éventuellement prêter sa voix aux protagonistes. Son interprétation peut donc prendre la forme tant d'un travail de marionnettiste - comme l'illustre la vidéo « Les petites bêtes » de l'équipe CFH Fan ${ }^{32}$, dans laquelle les mouvements des personnages servent à mettre en images une chanson de l'humoriste François Perusse -, que d'une performance de doubleur, comme c'est notamment le cas dans la vidéo « Des vacances qui commencent bien! $»$ du groupe BangBang $\mathrm{TV}^{33}$.

Illustration 1 : Capture d'écran du machinima « Des vacances qui commencent bien ! » réalisé par le groupe BangBang TV

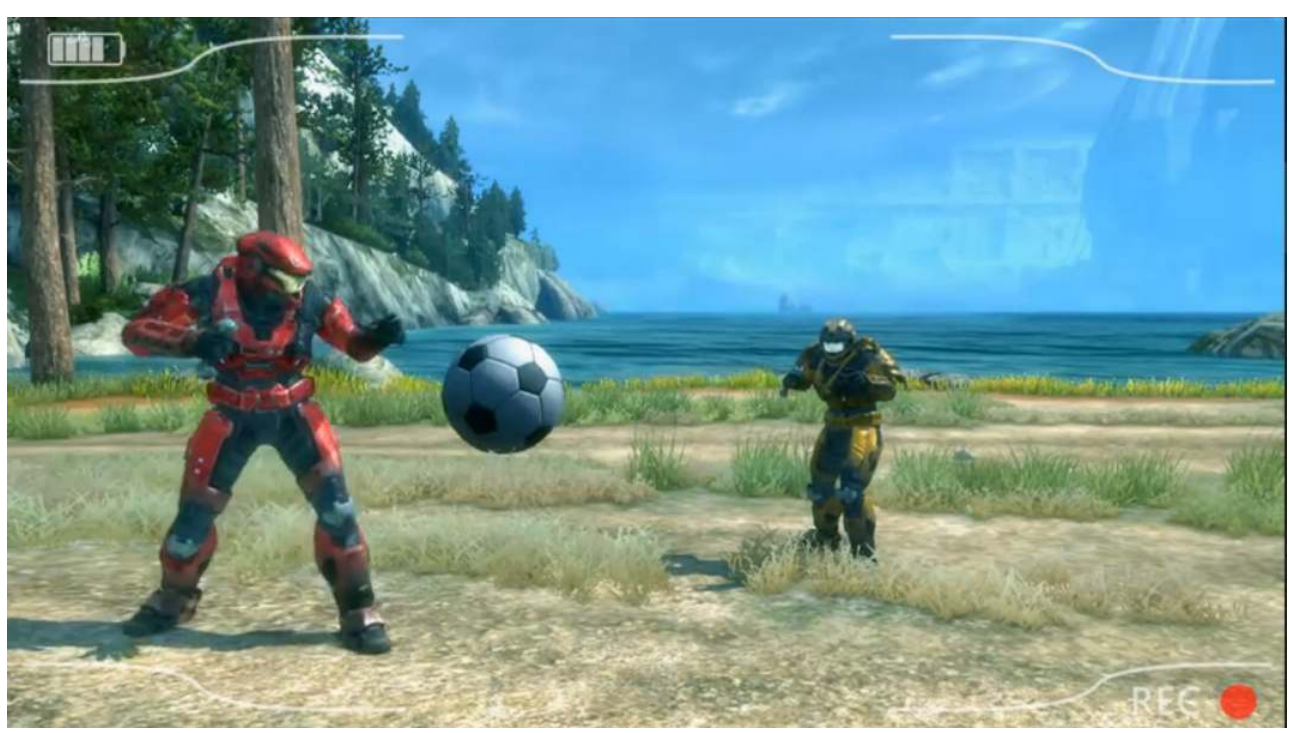

On passe donc ici du « jeu » au sens ludique au « jeu » au sens théâtral. Les machinimakers du groupe ILL Clan ont, d'ailleurs, poussé cette logique au maximum en utilisant le jeu vidéo pour mettre en place des spectacles d'improvisation diffusés en temps réel, comme dans leur vidéo "Common Sense Cooking with Carl the Cook», qui résulte d'une improvisation performée lors du Festival du film de Floride en $2003^{34}$. En outre, s'il est incontestable que cette pratique est profondément imprégnée des codes du cinéma ${ }^{35}$, les joueurs-producteurs ont plutôt tendance à se présenter comme des marionnettistes que comme des réalisateurs : « traditionally animation was a matter of scripting and editing, with each movement painstakingly built frame by frame. In Machinima, movement is created in real time by a performer who is part puppeteer and part actor » (interview de 
Chris Burke, créateur de la série This Spartan Life $e^{36}$ ); "we are using the video game characters like puppets that we manipulate and then record in real time. But instead of pulling strings, we use a video game controller to move them through space " (témoignage de Michael Burns, membre du groupe Rooster Teeth Productions ${ }^{37}$ ). Cette superposition de rhétoriques différentes est ce qui, selon le chercheur Michael Nitsche (2007), rend difficile la description du machinima à partir d'un discours disciplinaire trop situé.

La performance en termes d'interprétation pourrait sembler totalement étrangère au domaine compétitif du speedrun : une étape essentielle, dans cette pratique, consiste en effet à déterminer quelle suite de manipulations permettra au joueur de terminer le jeu en un temps record. La "partition » constituée par cet enchaînement d'actions tolère donc a priori peu l'improvisation ou l'interprétation personnelle - à moins que celles-ci n'entraînent un gain de temps. Pourtant, la mise en scène constitue également une valeur défendue par les sites qui structurent la pratique : ceux-ci définissent le speedrun comme un spectacle dont le caractère impressionnant ne dépend pas uniquement de la vitesse d'exécution, mais aussi de l'originalité de la performance. En témoigne cette déclaration des administrateurs du site TASVideos adressée aux praticiens: «be interesting. If you have the choice, try to do things in a more impressive way than the easy way. If enemies are hard to kill, kill them. If an object is hard to miss, miss it. If you can avoid taking damage without wasting time, do so $\aleph^{38}$. Dans le même ordre d'idées, les administrateurs du site Speed Demos Archive se réservent le droit de refuser une vidéo qui établirait un nouveau record de temps mais qui démontrerait une "pauvre qualité de jeu»: «note that it is possible for a run to be faster than the previous run in the same category and still be rejected. Perhaps the runner used a faster system, but otherwise the play quality was worse $\aleph^{39}$. En intégrant la qualité de l'interprétation dans leurs critères de publication, ces sites de référence construisent donc une norme qui vient contraindre une partie de la production. Ainsi, pour exemple, lorsqu'un jeu impose un temps d'attente au speedrunner (lors des passages en scrolling forcé ${ }^{40}$, notamment), celui-ci tentera généralement de faire effectuer à son avatar des actions visuellement drôles ou étonnantes ${ }^{41}$.

Enfin, le modding constitue, lui aussi, une performance interprétative: il suppose un réagencement plus ou moins créatif des éléments du jeu, qui peut répondre à des préoccupations techniques, tel le manque de convivialité de l'interface dans The Elder Scrolls V: Skyrim ${ }^{42}$, ou narratives, à l'image du mod «Alternate Start - Live Another Life »" qui offre au joueur de choisir où et dans quelles circonstances il commencera l'aventure de Skyrim - choix qui aura des conséquences sur tout le déroulement du jeu. Si l'originalité ou la pertinence de la réinterprétation proposée par un mod ne constituent pas des conditions déterminant la production, il s'agit tout de même de critères d'évaluation régulièrement présents dans les discours des praticiens. Ainsi les commentaires du mod «Falskaar » - qui ajoute au jeu une nouvelle contrée - saluent-ils la cohérence des modifications par rapport à l'univers : «I really like it, especially the new music. It feels like it belongs in skyrim, while also having a better atmosphere to it all around »; "this is overall my favorite mod for skyrim. The quests, the lore, it's all so unique, and I adore the work done. Such a lore-friendly mod $»^{44}$. À l'inverse, le manque de créativité peut aussi soutenir une critique : "mapping is amongst the best I have seen, though I am not sure about the choice you made about Falskaar's geography, it is a rectangle divided into 3 parts by mountains. It could have been lakes, valleys, dunno, 
something more creative $»^{45}$. La nature de l'interprétation proposée par le mod se distingue toutefois en ce que la "partition » elle-même s'y trouve être modifiée : contrairement aux autres formes de détournement, les mods ne laissent pas l'œuvre originale intacte puisqu'ils modifient le code source du jeu, ne serait-ce qu'en s'y agrégeant. On a donc affaire ici à un cas concret de transformative play, toujours selon Salen et Zimmerman (2004): le jeu en tant qu'activité interprétative est susceptible d'engendrer un nouveau dispositif.

\section{La lecture comme performance}

Si la production de détournements est analogue au jeu en ce qu'elle constitue, elle aussi, une performance, cette parenté peut s'étendre à la consommation des mêmes détournements. En effet, la forme fragmentée et la profonde intertextualité des œuvres dérivées ont pour effet de construire un «lecteur modèle » $(\mathrm{Eco}, 1985)$ très particulier, doté de la compétence nécessaire - en ce qui concerne les fanfictions et les machinimas pour reconnaître les références, citations ou variations présentes dans les textes, et dans le cas des speedruns et des mods ${ }^{46}$ - pour comprendre les techniques employées ou les altérations apportées au système du jeu. Par exemple, la vitesse de déplacement atteinte dans le TAS du jeu Sonic the Hedgehog $2^{47}$ ainsi que les glitchs exploités représentent un réel obstacle pour la lecture (le déroulement de l'écran est parfois incapable de suivre les mouvements de l'avatar): ces contraintes invitent donc le récepteur à adopter une lecture circulatoire, voyageant entre les commentaires du tool-assisted speedrunner (dans lesquels celui-ci détaille les techniques employées), la vidéo détentrice du précédent record, avec laquelle il établit de régulières comparaisons, et une seconde version du TAS où la caméra a été piratée pour rester centrée sur le personnage de Sonic ${ }^{48}$. Dans ce cas de figure, la lecture est plus que jamais envisagée comme une performance active.

Illustration 2 : Vidéo comparant les deux versions du TAS de Sonic the Hedgehog 2

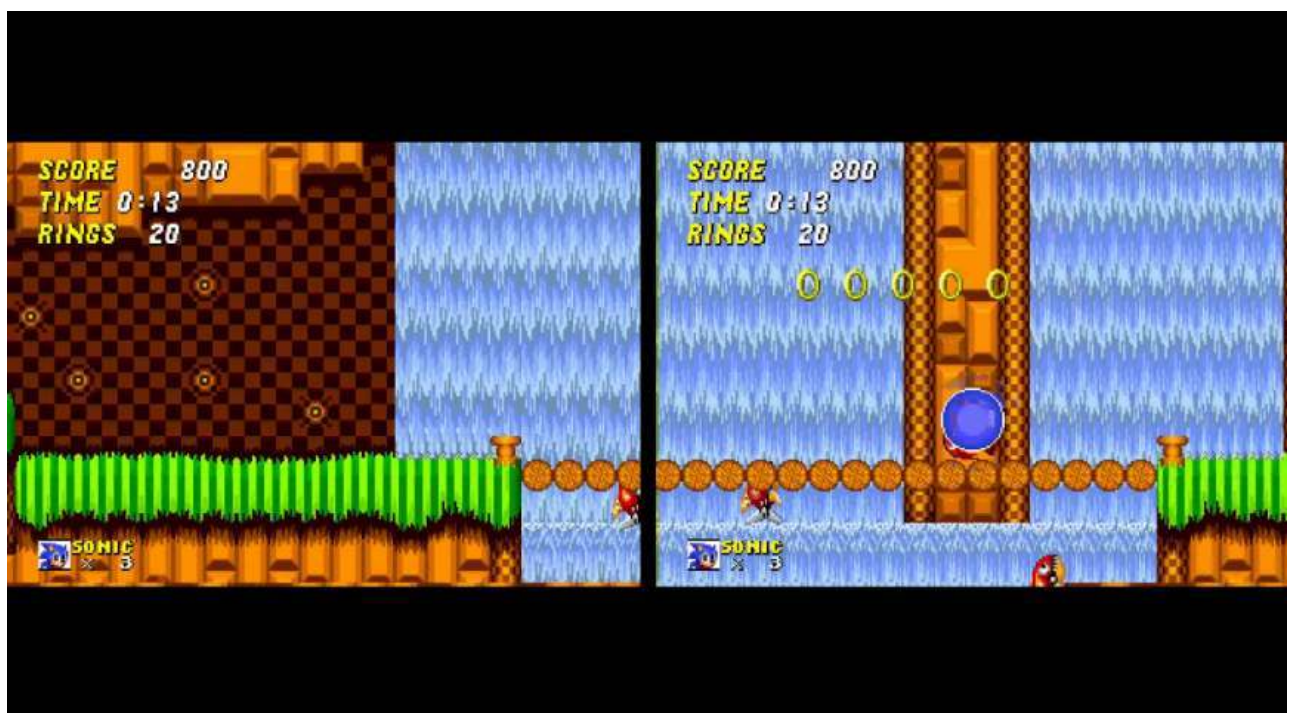

L'écran de gauche ne suit pas les mouvements de l'avatar tandis que, dans l'écran de droite, la «caméra » est fixée sur lui

Notons d'ailleurs que la participation des consommateurs est aussi évaluée à la manière d'une performance compétitive, en particulier à travers le nombre de commentaires qu'ils postent sur les sites consacrés. Ce nombre donne parfois lieu à une hiérarchisation 
des fans, comme en témoigne la figure du nouvel arrivant (parfois appelé «noob»), souvent objet de railleries: ainsi, le forum du site TASVideos mentionne le nombre de commentaires publiés par les participants en dessous de leur avatar, ce qui le fait donc réapparaître à chacune de leurs prises de parole; de même, sur Nexus (modding), ce nombre s'accompagne d'un «titre " mesurant l'implication du membre ("stranger », " newbie ", "fan », etc.) ; sur le site du groupe de machinimakers Rooster Teeth Productions, c'est l'investissement dans la communauté qui est récompensé par l'obtention de « points de karma » donnant accès à des forums réservés. Enfin, si la participation n'est pas aussi directement hiérarchisante dans le domaine des fanfictions, les lecteurs qui réagissent sous la forme de commentaires sont chaudement remerciés ${ }^{49}$, tandis que ceux qui lisent sans se manifester sont catalogués sous l'étiquette péjorative de «lecteurs fantômes " ${ }^{50}$. La valorisation de la participation est manifeste sur les forums de Fanfiction.net, où l'on trouve des sujets tels que : «FIC - Front d'incitation aux commentaires» («vous trouvez que les lecteurs ne laissent pas assez de commentaires? Vous voulez vous engager officiellement à en laisser davantage ? Rejoignez-nous, les commentaires vaincront ! ${ }^{51}$ ) ou «APAGCPMDEO » ("L'Association Pour Aider les Gens qui Croient qu'ils n'ont Pas de Mains à Découvrir qu'ils En Ont ! Militons pour le Droit à la Review ! Et ouvrons les yeux aux méchants SansMains Radins qui ne laissent jamais de reviews ! $\aleph^{52}$ ). D'autres sujets de discussion recensent des conseils ou des ressources dans le but d'aider les auteurs à obtenir davantage de commentaires ${ }^{53}$.

En somme, le détournement d'un jeu vidéo, comme la partition, n'a que peu de sens en dehors de son actualisation par un public: "the distinctions between authors and readers, producers and spectators, creators and interpretations will blend to form a reading-writing continuum, [...] each helping to sustain the activities of the others " (Levy, 1987, cité par Jenkins, 2006 : 144). La consommation de ce type d'œuvre offre la possibilité au lecteur d'entrer dans une pratique à laquelle il est encouragé à participer. Cette sollicitation - qui rappelle le concept d' 'impératif d'action $»^{54}$ développé par Genvo pour le jeu vidéo - est, d'ailleurs, inscrite dans la forme des réappropriations, comme le démontrera le point suivant.

\section{Affordance et interactivité}

Pour satisfaire à l'« impératif d'action » qui le caractérise, le jeu vidéo intègre, dans son dispositif même, des incitations à l'usage: il exhibe ses possibilités d'action à travers divers «marqueurs pragmatiques» (Genvo, 2011: 76). Ce phénomène peut être éclairé par le concept d'«affordance» (Gibson, 1979), qui désigne la capacité de certains dispositifs à s'offrir à nous pour nous faire agir (la poignée de porte appelle à être saisie, etc.). Ainsi, dans le cas du jeu vidéo, l'utilisateur est incité à explorer toutes les opportunités qui s'offrent à lui : le jeu se définit comme "l'exercice des possibles » (Genvo, 2011: 72).

Or les pratiques de réappropriations elles-mêmes sont le fruit de cet exercice et maintiennent de surcroît une affordance à portée ludique. Leur statut de « work in progress " (Hellekson et Busse, 2006: 6-7) rend ces productions non pas intouchables, comme ce serait le cas des œuvres culturelles «traditionnelles ", mais hautement manipulables. Les détournements demeurent toujours des textes tronqués, où le plaisir du processus de production a au moins autant de valeur que le produit fini, et où «the recipients of the open work are invited to fill in the blanks" (Jenkins, 2006: 145). En somme, les 
réappropriations de jeux vidéo ont également en commun avec leur médium d'origine leur dimension interactive, dont voici quelques « marqueurs ».

\section{Les fanfictions : un processus dynamique}

effet, si ces textes peuvent atteindre une longueur considérable ${ }^{55}$, ils n'en sont pas moins publiés progressivement, par bribes constamment recorrigées et mises à jour - mode d'écriture qui n'est rendu possible que par l'utilisation d'Internet comme support. L'importance secondaire accordée à la continuité de l'œuvre se marque notamment dans le recours fréquent aux genres tels que le one-shot (histoire ne comportant qu'un seul chapitre) ou le drabble (texte court, d'environ cent mots), ainsi que dans l'aspect fragmenté des textes: ceux-ci sont généralement divisés en chapitres pouvant euxmêmes être fractionnés en diverses sections ${ }^{56}$. Cette perte de la linéarité affecte directement les modalités de la lecture, la rendant hypertextuelle : la consultation d'une fiction nécessite de circuler entre différents chapitres, différentes histoires (de nombreux textes étant inachevés) et même différents sites - une tendance récurrente chez les « fanficcers » étant de renvoyer à des listes de musiques dont ils conseillent l'écoute durant la lecture ${ }^{57}$. C'est donc le parcours individuel de chaque lecteur qui vient conférer une continuité à des bribes de contenu entre lesquelles les auteurs n'établissent pas nécessairement de lien. Pour illustration, dans l'introduction de la fiction " Axel et Roxas dans cent thèmes ", l'auteure affirme, dès le deuxième chapitre que "ce recueil va être composé de drabbles qui ne suivront pas d'ordre chronologique particulier et qui pourront se trouver en totale contradiction les uns avec les autres. Il faut les prendre séparément les uns des autres et non comme un ensemble $»^{58}$. Le morcellement du texte fait donc de la lecture une activité de reconfiguration.

autre part, le temps d'attente qui peut séparer les différentes publications invite les lecteurs à imaginer eux-mêmes cette suite qui tarde à venir, voire à se lancer dans l'écriture : ainsi l'auteure Ariani Lee signale-t-elle, dans l'introduction d'un de ses textes, avoir été incitée à l'écriture par la lecture d'une autre fanfiction, à laquelle elle emprunte certains éléments : « remerciements à GAIL LLD, qui m'a donné envie d'écrire une fic avec Reno et de m'essayer au genre humoristique [...] $\aleph^{59}$. Il n'est d'ailleurs pas anodin que les jeux ayant suscité le plus de réécritures (Kingdom Hearts, Pokémon, Final Fantasy VII,...) aient connu plusieurs actualisations « officielles », sur différents médias (roman, manga, film d'animation,...), et séparées dans le temps: les espaces laissés vides entre les différents opus ont pu encourager les amateurs à prendre le relai. Cette dynamique interactive de la lecture fait de la fanfiction un processus sans fin puisque, "when the story is finally complete and published, [...] the work in progress among the creators shifts to the work in progress among the readers, and a whole new level of discourse begins that provides engagement and both positive and negative feedback » (Hellekson et Busse, 2006 : 7).

Outre l'espace de création qu'elles offrent, les fanfictions incitent également à l'action par des marques plus concrètes. Le système de la bêta-lecture, mentionné plus haut, constitue une démonstration éclairante du pouvoir qui est conféré au lecteur dans la communauté. Ces amateurs qui croisent les fonctions de lecteur, de critique et d'éditeur (Karpovich, 2006) affectent directement les textes qui leur sont confiés avant même leur publication et sont chaleureusement remerciés au sein du produit final : « note de l'auteur 3 - la plus 
importante !!! : Cette fic a été relue, corrigée et commentée (le soleil de mes relectures) par ma bêta-lectrice et amie Lily[u]. [...] Merci pour ton travail, pour tes commentaires (je ne pourrai plus jamais écrire sans ça!) et ta présence $»^{60}$; «merci beaucoup à Nayru25 d'avoir 'supervisé' cet OS [one shot] !=D Tes avis m'ont beaucoup aidée ${ }^{\wedge}$ [...] Et je te remercie aussi pour m'avoir aidée à trouver un titre $=) »^{61}$.

Cette superposition d'instances créatrices n'est pas problématisée mais reconnue comme partie intégrante du système. Les textes (voire les différents chapitres) se terminent d'ailleurs souvent sur un mot de l'auteur invitant les lecteurs à donner leur avis ou à apporter des corrections : «j'ai créé un sondage sur mon profil, parce que j'aimerais bien connaître votre avis quant aux personnages de la fanfic, pour savoir lesquels je dois plus développer, ou ceux qui ne méritent que de rester encore en retrait... Je compte sur vous pour y jeter un œil, si vous avez le temps. =) Merci d'avance ! $\wedge^{\wedge} \|^{62}$; « voilà le résultat, dites-moi ce que vous en pensez (bonne ou mauvaise, une critique est toujours bonne à recevoir ;)) et si vous trouvez que cette fic a de l'avenir ${ }^{63}$.

\section{Le potentiel interactif du machinima}

Les machinimas apparaissent comme le résultat direct de l'affordance propre au jeu vidéo puisqu'ils ont été rendus possibles par l'exploitation d'opportunités inscrites dans le game d'origine et ce, dès le jeu DOOM, avec notamment la possibilité d'enregistrer la partie in extenso. D'autre part, bien qu'il soit aujourd'hui possible d'utiliser l'œuvre vidéoludique comme un simple moteur d'animation, rares sont les auteurs de " machinimas de fans ${ }^{64}$ qui renoncent à toute référence à l'univers du jeu : non seulement l'univers graphique y est évidemment répété, mais les créations en «reprennent les codes en truffant leurs contenus de références à leur histoire " (Georges et Auray, 2012b: 5 version numérique). La série Arby 'n' the Chief du machinimaker Digitalph33r, par exemple, met en scène deux figurines de la franchise Halo jouant à divers opus du jeu éponyme (les vidéos alternent donc entre des séquences tournées au sein du jeu et des passages filmés en prises de vue réelles, dans lesquels apparaissent les figurines). La pratique ludique de ces deux personnages - qui incarnent respectivement les archétypes du «bon » et du " mauvais » joueur - permet à l'auteur de représenter de façon scénarisée des situations typiques du jeu multijoueurs ${ }^{65}$. Dans le même ordre d'idées, la série Red vs. Blue s'élabore autour d'un principe fondamental du mode multijoueurs de Halo: la lutte entre deux équipes, les bleus et les rouges, pour s'emparer du drapeau adverse - l'inscription de ce point de gameplay dans un récit permettant aux auteurs d'ironiser sur son absurdité. Ces régulières « citations ludiques » peuvent donc être vues comme autant d'invitations au jeu. 
Illustration 3 : Passage de la série Arby ' $n$ ' the Chief mettant en scène les figurines en prises de vue réelles

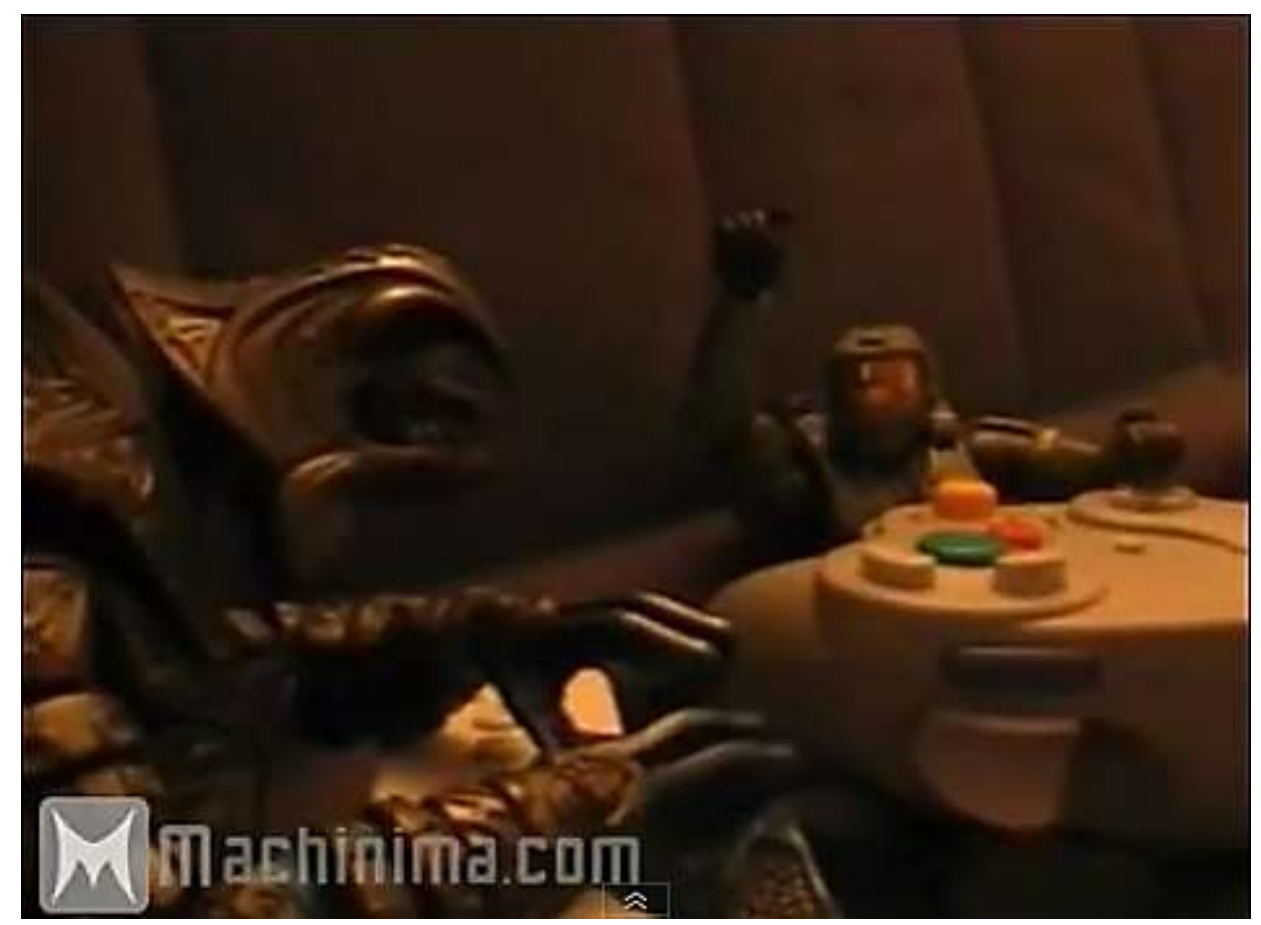

En outre, la réalisation de machinimas n'échappe pas à la dynamique interactive décrite précédemment au sujet des fanfictions : les interventions et commentaires des récepteurs sont souvent sollicitée ${ }^{66}$, immédiats (en raison du mode de diffusion des vidéos, via des plateformes telles que YouTube) et effectifs, selon certains producteurs : « on the Internet, you can tell people whatever you want, and you can ask for whatever you want. The audience dictates a lot ${ }^{67}$. Un exemple de cette effectivité peut être trouvé dans l'épisode «Conflict - Part 1 » de la série Arby ' $n$ ' the Chief, présentée ci-dessus, où l'auteur profite du générique final pour lancer un sondage concernant l'avenir de deux personnages secondaires qui avaient été critiqués par les spectateurs : « I may not have responded, but I have read your pleas and hate mail regarding the new characters. Perhaps they were a mistake. I'm leaving their fate in your hands. To vote for your desired outcome, log on to digitalph33r.blogspot.com, and in the top-right corner, there will be a poll where you may vote for your desired outcome in the next episode [...] $\|^{68}$.

Dans certains cas extrêmes, les espaces de production et de réception ne sont même plus distingués. Ainsi, la série This Spartan Life, développée par Chris Burke, se présente sous la forme d'un talk-show situé dans le jeu de tir Halo 2 : un animateur y reçoit des intervenants qui prennent la parole sur différents sujets d'actualité au milieu d'un champ de bataille multijoueurs. Les attaques constantes des joueurs ordinaires (à qui il arrive fréquemment d'ôter la vie d'un intervenant ou du présentateur) teintent alors la production d'une dimension aléatoire qui contribue sans aucun doute à sa portée humoristique.

\section{" Jouer au modding »}

L'incitation à l'action contenue dans le mod est de nature évidente, puisqu'il s'agit d'un outil pour jouer différemment. Néanmoins, l'affordance propre à ces altérations de l'œuvre vidéoludique ne se contente pas d'encourager à (re)jouer au jeu, mais invite aussi 
à «jouer au modding ». En effet, la seule installation de mods implique de maîtriser certaines compétences (ne serait-ce que pour éviter de les faire entrer en conflit et d'entraîner le dysfonctionnement $\mathrm{du}$ jeu): leur consommation demande donc un apprentissage qui constitue déjà une entrée dans la pratique. Ainsi la description du mod "RaceMenu ${ }^{69}$ commence-t-elle en détaillant les différents pré-requis nécessaires à son fonctionnement, tels l'installation d'un utilitaire et d'un module complémentaire, puis poursuit en mentionnant les mods avec lesquels « RaceMenu » n'est pas compatible et en recensant les divers problèmes pouvant être rencontrés par le joueur. Dans le même ordre d'idées, les utilisateurs de mods peuvent avoir une fonction de bêta-testeurs et participent à améliorer les productions, notamment en rapportant les anomalies rencontrées : parmi les commentaires du mod intitulé « Frostfall - Hypothermia Camping Survival », par exemple, on trouve plusieurs messages s'annonçant comme des rapports de dysfonctionnements ( bug report ») et détaillant, selon un schéma précis, un problème rencontré dans la version modifiée du jeu ${ }^{70}$.

Par ailleurs, le succès de la pratique du modding a poussé l'industrie vidéoludique à intégrer l'intervention des joueurs dans sa structure même: certains jeux mettent à disposition des "kits de développement» permettant aux utilisateurs d'éditer leurs propres niveaux et de les diffuser ; c'est le cas, notamment, de The Elder Scrolls V: Skyrim, de TrackMania et de la plupart des FPS. En outre, le fait que le modding puisse, dans certains cas, constituer le fondement du gameplay d'un jeu (dans des œuvres telles que Minecraft ou Little Big Planet, qui s'apparentent à des jeux de construction et où une grande partie du contenu est produite par les joueurs), manifeste la nature profondément ludique de ce type de création : les développeurs ont pris conscience de la "ludogénéité " (Vial, 2012) intrinsèque à l'acte de programmation.

\section{Le speedrun : une « construction abductive »}

41 Dans la pratique du speedrun, les marques d'affordance incitant le spectateur à s'engager dans le jeu s'inscrivent tant dans la forme des vidéos que dans le fonctionnement de la communauté. Dans la forme des vidéos, tout d'abord, car celles-ci sont accompagnées d'un texte descriptif expliquant les différentes techniques qui ont rendu le record possible $^{71}$ ou même, si l'auteur les connait, les passages de la vidéo qui pourraient être améliorés. La présentation du speedrun de Dragon Warrior, par exemple, se termine sur ces conseils : « in closing, here are some things that an improved Dragon Warrior run could try : killing four Metal slimes to reach level seven, one segment each (yes, this is possible, just very hard), saving around forty minutes; [...] and killing the Dragonlord at level seventeen using SLEEP [...], saving twenty-five minutes. In all, you could bring the time down to about $3: 30 »^{72}$.

Loin de conserver secrètement leurs astuces dans une optique - pourtant présente - de compétition, les membres de cette communauté reconnaissent volontiers la dimension collective de leur pratique (il est nécessaire de collaborer pour optimiser les performances individuelles) et la nature éphémère de leur propre record. Ainsi l'actuel détenteur du meilleur temps sur le jeu Super Mario Bros fait-il l'historique, dans le commentaire de sa vidéo, des différents records qu'il a surpassés (les siens y compris) ${ }^{73}$. 


\section{Author's comments: \\ Speedrun History: \\ February 26 2004, 5:11, Scott Kessler \\ April 23 2004, 5:10, Scott Kessler \\ October 9 2004, 5:07, Trevor Seguin \\ November 7 2004, 5:06, Trevor Seguin \\ September 14 2006, 5:05, Scott Kessler (5:08 by Twin Galaxies) \\ April 10 2007, 5:00, Andrew Gardikis \\ December 24 2010, 4:59, Andrew Gardikis \\ December 15 2011, 4:58, Andrew Gardikis (current)}

Ensuite, l'invitation à agir se marque également dans le fonctionnement des échanges entre praticiens (sur les forums et dans les commentaires des vidéos), souvent empreints d'une dimension pédagogique: «many of the insights collected during the making of these runs is documented on our resource pages, allowing new players to quickly catch up to our experts", signalent les administrateurs du site TASVideos ${ }^{74}$. La communauté collabore effectivement dans la constitution d'importantes bases de connaissances et de conseils sur les jeux détournés ${ }^{75}$. L'inclusion des spectateurs dans la pratique passe, en outre, par les nombreux débats qui se développent sur les forums et qui, à l'image du sujet «Debate : allowed or not? $»^{76}$, mettent en question les règles et les valeurs de la pratique. Selon Andrés Delikat, administrateur de TASVideos, «the community has a big impact on the rules, and those rules change overtime as the concept of TAS evolves ${ }^{77}$.

Au-delà de l'incitation à participer, les caractéristiques du speedrun font de cette activité une «construction abductive» (au sens de Perriault et Sellah, qui appliquent la logique piercienne au jeu vidéo), en ce qu'elle « conduit le joueur à considérer une action possible comme une hypothèse à vérifier» (Perriault et Sellah, 2012: 73). En effet, le speedrun modifie radicalement la manière de jouer à l'œuvre originale puisque les objectifs traditionnels sont négligés au profit de la recherche des bugs exploitables, ces fameux glitchs. En plus de répondre à l'exigence de rapidité propre à la pratique, le TAS du jeu de réflexion Programme d'entraînement cérébral du Dr Kawashima: Quel âge a votre cerveau $?^{78}$ s'attelle, par exemple, à démontrer la possibilité de tromper la console de jeu. En effet, le défi proposé par cette œuvre vidéoludique consiste à résoudre une série de calculs et à en écrire la réponse à l'aide d'un stylet sur l'écran tactile de la Nintendo DS. Or les outils de l'émulation permettent ici au tool-assisted speedrunner de résoudre les opérations mathématiques en inscrivant non des chiffres, mais des dessins dans lesquels la console reconnaît tout de même la bonne réponse. 
Illustration 5 : TAS du jeu de réflexion Programme d'entraînement cérébral du Dr Kawashima : Quel âge a votre cerveau?

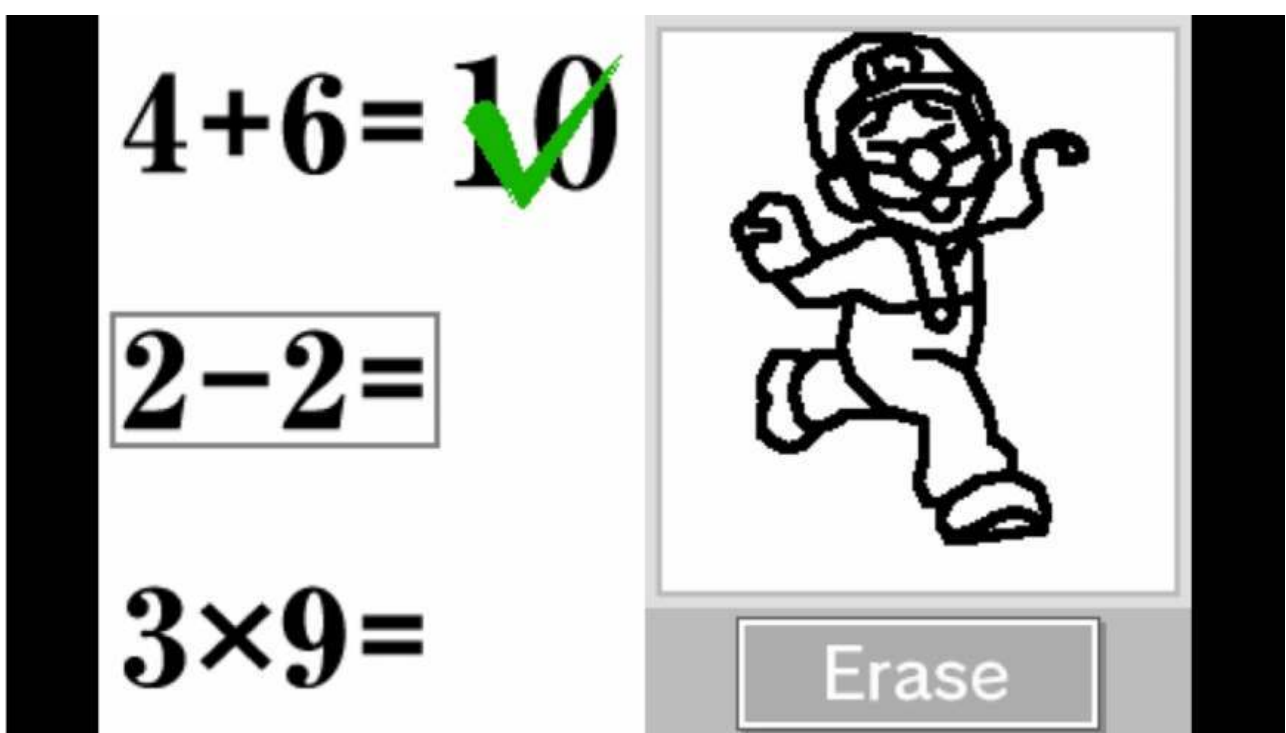

Une telle contrainte modifie donc le gameplay du jeu et constitue une étape supplémentaire dans la maîtrise du système, puisque l'objectif n'est plus de calculer rapidement mais de proposer la réponse la plus inattendue possible. Ce nouveau mode de réception rejoint les conclusions du philosophe Azuma, pour qui le public postmoderne consomme les œuvres culturelles suivant une double logique : d'une part en trouvant du plaisir dans une lecture de surface, non distanciée, d'autre part en aspirant constamment à s'emparer du système des œuvres, c'est-à-dire de la macrostructure qui a présidé à leur construction, "pour extraire et s'approprier les informations avant leur mise en forme dans une image ou une scène [et] utiliser ce matériau dans la construction d'un autre produit » $(2008: 130)$.

À travers ces différentes figurations de l'interactivité au sein des détournements se fait jour une autre caractéristique propre aux détournements de jeux vidéo: ceux-ci ne cherchent pas à faire oublier le médium qui les supporte ni celui dont ils s'inspirent. Ils ne s'affichent pas comme des œuvres autonomes mais s'affirment, au contraire, comme des variations. En référant constamment au jeu par le biais de citations plus ou moins explicites, ils rappellent le récepteur à son statut premier de joueur.

\section{Auteur ou joueur?}

On l'a vu, l'avènement d'Internet a notamment eu pour effet de doter les amateurs d'outils et de savoir-faire leur permettant d'éventuellement atteindre un niveau comparable à celui des experts. Dans ce contexte émerge une nouvelle figure : celle du "pro-am ", un fan qui impose des standards professionnels à son activité (Flichy, 2010). Toutefois, malgré cette expertise, les amateurs concernés ne semblent pas tous chercher à se substituer aux véritables professionnels : un écart demeure, dont l'explication n'est pas à chercher dans une différence de compétence mais bien dans « une autre forme d'engagement dans les pratiques sociales » (Flichy, $2010: 12$ ). 


\section{Des pratiques amateurs}

48 jeux vidéo. En effet, malgré les exigences techniques et créatives que ces pratiques requièrent, les discours tenus par leurs auteurs demeurent empreints d'un refus de prendre leur activité "au sérieux». Loin de revendiquer un statut d'artiste et/ou de professionnel, ces joueurs semblent généralement déconstruire leur éventuel ethos d'auteur - au sens fort du terme - par divers moyens. On a déjà mentionné, par exemple, la dimension collective de la création, dans le cas du speedrun ou des fanfictions: la collaboration qui est à la base de ces pratiques fait ainsi obstacle à l'identification d'une figure unique de créateur. Dans le cas précis des fanfictions, «[...] the practice of beta reading represents a further refutation of the idea that individuals or groups can claim sole intellectual ownership over the texts [...] 》 (Karpovich, 2006 : 176).

Les discours tenus par les machinimakers ou les moddeurs reconnus - lorsqu'ils n'ignorent pas tout simplement la dimension artistique que certains prêtent à leurs créations s'attachent également à construire une posture d'amateur. Ainsi, malgré les récompenses qui leur ont été octroyées (lors des remises de prix annuelles de l'International Academy of Web Television ou de l'Academy of Machinima Arts \& Sciences) et malgré une certaine professionnalisation (il s'agit de studios dont les membres sont rémunérés pour leurs productions), les machinimakers de Rooster Teeth et Bong + Dern Productions se bornent, sur leur site respectif, à décrire leur projet en des termes purement humoristiques et romancés. En témoigne la page de présentation de la série This Spartan Life ${ }^{79}$, dans laquelle l'auteur réel, Chris Burke, n'est mentionné qu'indirectement, à travers le personnage qu'il incarne : «Damian Lacedaemion is not your average talk show host. He lives inside an online game where daily existence is a struggle against overwhelming odds (and other, much better players.) So he has taken it upon himself to create a little oasis of sanity where he can talk to people who aren't bent on his destruction ${ }^{80}$. De même, l' « histoire de Rooster Teeth », présentée sur le site du studio, se termine en ces termes : «the group reunited in a flurry of activity. Plans were made, schemes were hatched, and just as they were settling on the idea of a Justin Timberlake fan site, Burnie remembered his little Halo movie. He knew there was only one way to convince Gus and Geoff (who were already drawing up scanning in their Timberlake pics), so he got them stinking drunk. They awoke the next day, hung over and strangely sore (but that's a story for another day), to see that Burnie had convinced them to sign their lives away for the promise of more Banana Daiquiris. [...] They were onboard. And the world was without the best Justin Timberlake site ever... ${ }^{81}$. Dans une perspective similaire, Georges et Auray (2012a : 8) mentionnent l'exemple de la réaction du machinimaker Kingrabbit qui, recevant le premier prix de l'Atopic festival 2011, a tourné en dérision cette tentative d'attribution d'une dimension artistique à son travail ${ }^{82}$.

50 Le domaine du tool-assisted speedrun pourrait constituer une exception à cette tendance, dans le sens où la pratique est prudemment qualifiée d'art par certains commentateurs (qu'il s'agisse de membres de la communauté ${ }^{83}$ ou d'observateurs extérieurs ${ }^{84}$ ). Néanmoins, si le potentiel artistique des vidéos produites est parfois affirmé, nous n'avons pas rencontré de discours où l'auteur revendique pour lui-même le statut d'artiste : la pratique est prise au sérieux, mais les praticiens se présentent généralement sur un mode distancié où l'ironie occupe une place importante, comme en témoignent les

RESET, 4 | 2015 
réponses fournies au sujet "Are Tool-Assisted Runs Conceptual Art?» sur le forum général du site $T_{A S V i d e o s}{ }^{85}$. Le processus de création des TAS va, d'ailleurs, à l'encontre de l'édification d'une figure de créateur inspiré, puisque le fait d'enregistrer les commandes actionnées dans un fichier séparé du jeu autonomise la performance par rapport à son auteur: le record existe indépendamment de celui qui l'a "programmé", et il est d'ailleurs courant que les tool-assisted speedrunners s'échangent les «partitions » de leurs prestations.

\section{Entre consécration et jeu}

Face à ces postures, les discours académiques et critiques produisent un important contraste. En effet, les observateurs externes à ces pratiques tendent plutôt à valoriser et à légitimer les pratiques de détournement en les rapprochant des structures artistiques traditionnelles, à l'image de cette intervention de Graham Leggat (critique de jeux vidéo et directeur des communications à la Film Society of Lincoln Center) dans un article du Wall Street Journal : «"the literary analog is absurdist drama [...]. It's [the machinima Red vs. Blue] truly as sophisticated as Samuel Beckett" ${ }^{86}$. Cette ambition s'exprime, d'autre part, dans la création d'institutions pourvoyeuses de récompenses et dans les tentatives concrètes de réappropriation artistique ou militante du phénomène. Le jeu de cuisine Cooking Mama, par exemple, a été détourné par une association pro-végétarienne dans le but de dénoncer la violence faite aux animaux (Cooking Mama: Mama kills animals ${ }^{87}$ ). Néanmoins, comme le soulignent Perriault et Sellah (2012), l'objectif sérieux du projet a totalement été négligé par les joueurs, qui l'ont plutôt reçu comme un jeu humoristique.

Illustration 6 : Écran-titre du détournement Cooking Mama : Mama kills animals

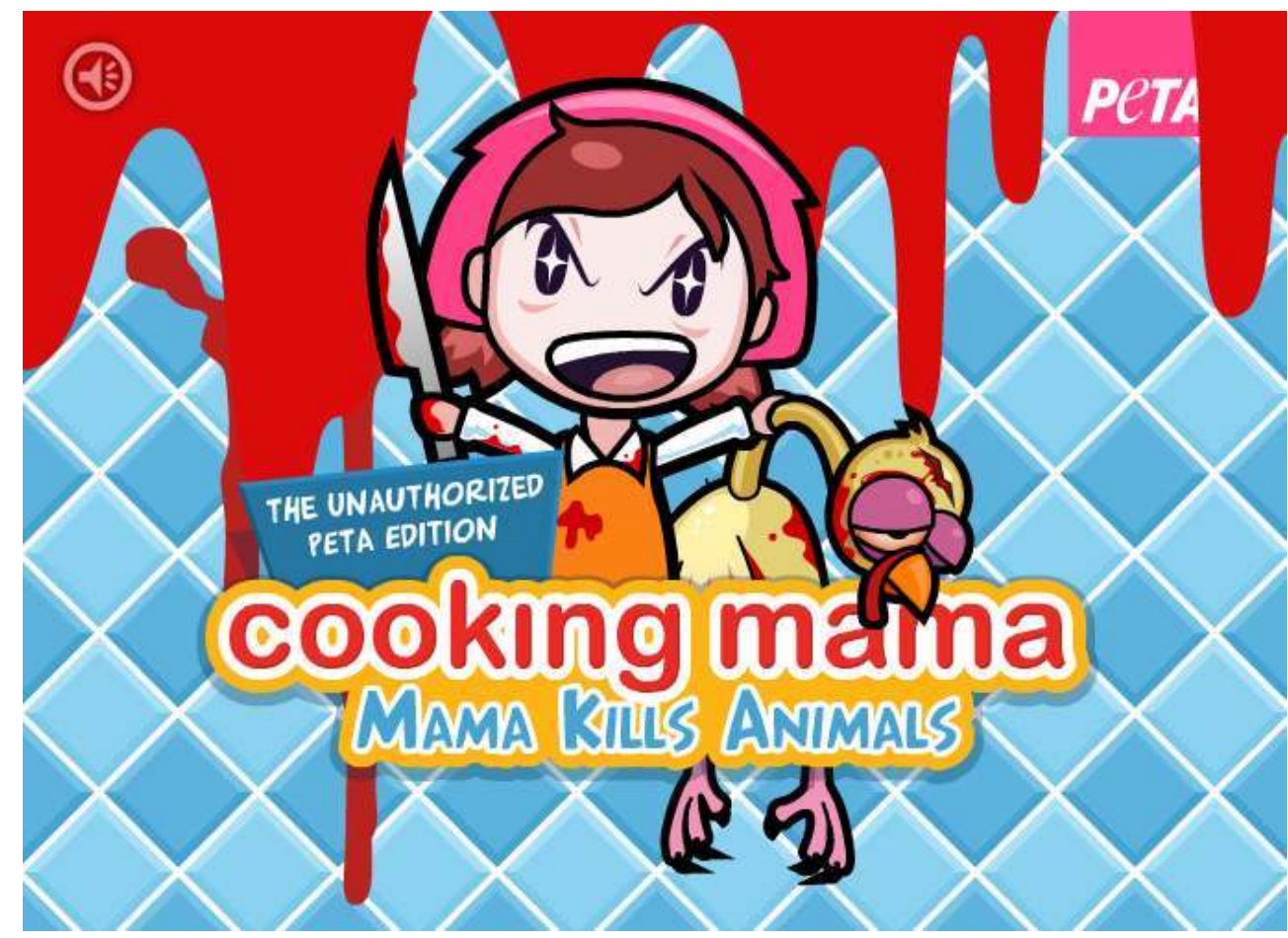

Ce dernier exemple illustre à quel point l'instrumentalisation des productions culturelles que sont les détournements peut faire obstacle à leur compréhension, celles-ci s'inscrivant dans une culture qui se revendique avant tout comme ludique. Comme le 
souligne Georges (2012), l'observation de ces phénomènes par les chercheurs fait entrer en conflit des communautés interprétatives différentes, ce qui pose un certain nombre de problèmes méthodologiques: comment, par exemple, formaliser l'œuvre sans tenir compte de cette revendication d'amateurisme? Selon cette perspective, les formes des détournements ne peuvent être envisagées indépendamment des discours élaborés par leurs auteurs car l'ethos qu'ils construisent (notamment à travers la revendication d'une appartenance à une culture ludique) entre dans la construction de la signification des œuvres.

Dès lors, la parenté entre le jeu vidéo et ses réappropriations peut également être établie comme une continuité de "posture » (cf. Meizoz, 2007 \& 2011) de la part des amateurs : en passant du jeu à la création, les joueurs ne cessent pas de se définir comme des joueurs. Leur constante autodérision peut apparaitre, au fond, comme le moyen de ne jamais sortir du « cercle magique » (Huizinga, 1950) : comme si leurs créations se devaient de rester sans conséquences dans le monde "réel», comme s'ils "jouaient " à être auteurs, cinéastes ou game designers. L'espace culturel dans son ensemble, ainsi investi par le play des joueurs, devient un vaste terrain de jeu dont ces derniers maitrisent et superposent les différents codes.

Si cette posture de "non-auteur" semble aller totalement à l'encontre des traditions culturelles et de l'idéal romantique (un auteur démiurge, un art isolé de la société et une esthétique de l'innovation), Éric Maigret ne manque toutefois pas de remarquer que ce même idéal n'a pas disparu des consciences, au point d'être à l'origine d'une «schizophrénie culturelle». Selon lui, les publics actuels «vivant de plus en plus de cultures médiatiques tout en éprouvant de grandes difficultés à s'éloigner de la norme lettrée » en viennent à développer un sentiment d'ironie et de culpabilité par rapport à leurs pratiques culturelles quotidiennes (Maigret, 2005 : 127). Il n'est, par conséquent, pas étonnant que ce mode distancié de consommation de la culture trouve une expression privilégiée dans le jeu et le second degré.

\section{Conclusion}

L'ambition panoramique de cette étude, puisqu'elle aborde différents points de passage entre le jeu et plusieurs pratiques qui se l'approprient, en limite nécessairement la précision: bien qu'interconnectés, les phénomènes observés sont en effet loin d'être homogènes. Les particularités narratives des fanfictions, le réagencement qu'elles proposent de l'univers fictionnel dont elles s'inspirent, par exemple, ne trouvent pas d'équivalent dans la pratique performative $\mathrm{du}$ speedrun. Chaque détournement demanderait donc une analyse ponctuelle qui en mettrait au jour les spécificités, les nuances et contradictions internes.

Plus précisément, cette première approche caractérisée par un mode d'observation indirect - c'est-à-dire abordant les pratiques de détournement à travers les traces qu'elles laissent dans les œuvres et dans leurs paratextes - pourrait être complétée par des études de terrain (entretiens, enquêtes quantitatives, etc.) qui éclaireraient le contexte de l'activité et les parcours cognitifs des praticiens. De telles enquêtes permettraient, en d'autres termes, de soulever une série de questions auxquelles le matériau ici interrogé ne permet pas de répondre, mais qui viendraient compléter la compréhension du détournement de jeux vidéo dans sa dimension collective et communautaire : les auteurs 
de détournements partagent-il un certain profil sociologique?; par quels biais et par quels acteurs sont construites les normes et valeurs décrites ci-dessus, qui constituent la vitrine de la pratique?; dans quelle mesure les praticiens s'y reconnaissent-ils et quel est leur influence effective sur la production?; les pratiques de détournement sont-elles effectivement vécues comme ludiques par ces créateurs ?; ou encore, quels sens donnentils à leur activité et comment l'articulent-ils (ou non) à leurs autres expériences de jeu ?

L'importance de tels questionnements complémentaires résiderait, selon nous, dans l'éclairage neuf que ceux-ci pourraient apporter aux problématiques contemporaines autour de l'importance grandissante des «cultures ludiques» (Wendling, 2002): l'avènement d'une culture participative amplifiée par la popularisation d'Internet (Raessens, 2005), le renouvellement des processus de création et de réception des œuvres culturelles à travers la généralisation de l'interactivité de leurs supports et, enfin, la propagation du ludique conceptualisée sous l'angle de la gamification (Deterding et al., 2011) ou de la «ludicisation» (Genvo, 2011). En effet, les manifestations décrites au sein de cet article sont des pratiques ludiques prenant le jeu pour support (elles « se jouent du jeu») et exportant certaines caractéristiques de l'expérience vidéoludique dans de nouveaux domaines (le texte littéraire, le cinéma, la vidéo, le travail de programmation). $\mathrm{Au}$ sein de ces mécanismes, Internet joue un rôle de liant : il prolonge le play hors de son espace premier et participe à établir, de cette façon, une porosité entre le ludique et des objets qui ne sont pas nécessairement «étiquetés» comme tels. Cette variété d'actualisations possibles du jeu permet aux amateurs de construire et de mettre en scène leur propre imaginaire autour du «jouer»(Genvo, 2008b). L'étude approfondie des détournements de jeux vidéo par les joueurs pourrait donc contribuer non seulement à caractériser les formes diverses que peut prendre l'expérience ludique, mais aussi à comprendre comment celle-ci s'articule avec les autres pans de leur culture.

\section{BIBLIOGRAPHIE}

AZUMA Hiroki (2008). Génération Otaku. Les enfants de la postmodernité, Paris, Hachette Littératures. BERRY Vincent (2008a). « Les communautés de pratiques : note de synthèse », Pratiques de formation. Analyses, 54, pp. 12-47.

BERRY Vincent (2008b). « Une "cyberculture" ludique, collaborative et paradoxale », MédiaMorphoses, 22, pp. 55-61.

CAMERON David \& CARROLL John (2011). « Encoding liveness: Performance and real-time rendering in machinima », in Lowood Henry \& Nitsche Michael (dir.) The Machinima Reader, Cambridge, MIT Press, pp. 127-141.

CoPPA Francesca (2006), «Writing bodies in space: Media fan fiction as theatrical performance », in Hellekson Karen \& Busse Kristina (dir.) Fan Fiction and Fan Communities in the Age of the Internet, Jefferson, McFarland, pp. 225-244. 
DAVALLON Jean, NOEL-CADET Nathalie \& BROCHU Danièle (2003). «L'usage dans le texte : les "traces d'usage" du site Gallica », in Souchier Emmanuël, Jeanneret Yves \& Le Marec Joëlle (dir.) Lire, écrire, récrire. Objets, signes et pratiques des médias informatisés, Paris, Éditions de la Bibliothèque publique d'information, pp. 47-90.

DETERDING Sebastian, DIXON Dan, KHALED Rilla \& NACKE Lennart (2011). « From game design elements to gamefulness: defining "gamification" », Actes de la conférence du festival MindTrek'11 : Envisioning Future Media Environments, Tampere, Finlande, pp. 9-15

DI CROSTA Marida (2009). Entre cinéma et jeux vidéo : l'interface-film. Métanarration et interactivité, Bruxelles, De Boeck.

ECO Umberto (1985). Lector in fabula : le rôle du lecteur ou la coopération interprétative dans les textes narratifs, Paris, Librairie Générale Française.

FLICHY Patrice (2010). Le sacre de l'amateur. Sociologie des passions ordinaires à l'ère numérique, Paris, Seuil.

FLON Émilie, DAVALLON Jean, TARDY Cécile \& JEANNERET Yves (2009). « Traces d'écriture, traces de pratiques, traces d'identités ", in Saleh Imad, Leleu-Merviel Sylvie, Jeanneret Yves, Massou Luc \& Bouhai Nasreddine (dir.) H2PTM'09. Rétrospective et perspective 1989-2009, Paris, Hermès-Lavoisier, Paris, pp. 181-191.

FRANÇOIS Sébastien (2007). «Les fanfictions, nouveau lieu d'expression de soi pour la jeunesse? ", Agora débats/jeunesses, 46, pp. 58-68.

GENVo Sébastien (2006). Le game design de jeux vidéo. Approche communicationnelle et interculturelle, Thèse de doctorat en Sciences de l'information et de la communication, Université Paul Verlaine, Metz.

GENVo Sébastien (2008a). «Caractériser l'expérience du jeu à son ère numérique : pour une étude du "play design" ", Communication présentée au colloque Le jeu vidéo : expériences et pratiques sociales multidimensionnelles, Québec.

GENVo Sébastien (2008b). « Comprendre les différentes formes de "faire soi-même" dans les jeux vidéo », Communication présentée au colloque Ludovia, Ax-Les-Thermes.

GENVo Sébastien (2011). «Penser les phénomènes de "ludicisation" du numérique : pour une théorie de la jouabilité ", Revue des sciences sociales, $\mathrm{n}^{\circ} 45,68-77$.

GENVo Sébastien (2012). « La théorie de la ludicisation : une approche anti-essentialiste des phénomènes ludiques ", Communication présentée au colloque Jeu et jouabilité à l'ère numérique, Paris. [Disponible en ligne sur LudoLogique.

GEORGES Fanny (2012). « Productions créatives issues du jeu vidéo et compétences communicationnelles ", communication présentée au colloque Jeu et jouabilité à l'ère numérique, Paris, 8 décembre 2012.

GEORGES Fanny \& AURAY Nicolas (2012a), « Approche sémiopragmatique de l'espace de communication des machinima », Revue des Interactions Humaines Médiatisées, 13 (1), pp. 3-36.

GEORGES Fanny \& AURAY Nicolas (2012b), « Les productions audiovisuelles des joueurs de jeux vidéo : entre contraintes industrielles et appartenance au champ artistique », Réseaux, 5 (175), pp. 145-173.

GIBSON James J. (1979). The ecological approach to visual perception, Boston, Houghton Mifflin.

Goodman Nelson (2005). Langages de l'art. Une approche de la théorie des symboles, Paris, Hachette. 
GREIMAS Algirdas Julien \& COURTÉs Joseph (1979), Sémiotique. Dictionnaire raisonné de la théorie du langage, Paris, Hachette.

HELLEKSON Karen \& BUSSE Kristina (dir.) (2006). Fan Fiction and Fan Communities in the Age of the Internet, Jefferson, McFarland.

HUIZINGA Johan (1950). Homo Ludens : A study of the play-element in culture, Boston, Beacon Press.

JENKINS Henry (1992). Textual Poachers : Television Fans and Participatory Culture, New York, Routledge.

JENKINS Henry (2006). Fans, bloggers, and gamers : Exploring participatory culture, Londres/New York, New York University Press,.

KAPLAN Deborah (2006). « Construction of Fan Fiction character through narrative », in Hellekson Karen \& Busse Kristina (dir.) Fan Fiction and Fan Communities in the Age of the Internet, Jefferson, McFarland, pp. 134-152.

KARPOVICH Angelina I. (2006). «The audience as editor: The role of beta readers in online fan fiction communities ", in Hellekson Karen \& Busse Kristina (dir.) Fan Fiction and Fan Communities in the Age of the Internet, Jefferson, McFarland, pp. 171-188.

LOWOOD Henry (2005). « Real-time performance : Machinima and game studies », IDMAa Journal, 1 (3), pp. 10-17.

LOWOoD Henry (2006). « High-performance play: The making of machinima », Journal of Media Pratice, 7 (1), pp. 25-42.

LOWOoD Henry (2008). « Found Technology: Players as Innovators in the Making of Machinima », in McPherson Tara (dir.) Digital Youth, Innovation, and the Unexpected, Cambridge, MIT Press, pp. 165-196.

MAIGRET Éric (2005). « Esthétiques des médiacultures », in Maigret Éric \& Macé Éric (dir.) Penser les médiacultures. Nouvelles pratiques et nouvelles approches de la représentation du monde, Paris, Armand Colin, pp. 123-144.

MEIZOZ Jérôme (2007). Postures littéraires. Mises en scène modernes de l'auteur, Genève, Slatkine Érudition.

MEIZOZ Jérôme (2011). La Fabrique des singularités. Postures littéraires II, Genève, Slatkine Érudition. NEWMAN James (2008). Playing with Videogames, Londres/New York, Routledge.

NITSCHE Michael (2007). « Claiming its space : Machinima », Dichtung Digital, 37 [en ligne], consulté le 14.12.2015. URL : http://dichtung-digital.de/2007/Nitsche/nitsche.htm.

PERRIAULT Jacques \& SELLAH Soraya (2012), « Le détournement des jeux informatisés comme œuvre artistique », in Fourmentraux Jean-Paul (dir.), L'Ère post-média, Humanités digitales et Cultures numériques, Paris, Éditions Hermann, pp. 71-85.

PICARD Martin (2007). « Machinima: Video Game As An Art Form? », Loading, Journal of the Canadian Game Study Association, 1 (1) [en ligne], consulté le 14.12.2015. URL : http:// journals.sfu.ca/loading/index.php/loading/article/view/17/20]

RAESSENS Joost (2005), « Computer Games as Participatory Media Culture », in Raessens Joost \& Goldstein Jeffrey (dir.) The Handbook of Computer Game Studies, Cambridge, MIT Press, pp. 373-388. SALEN Katie \& ZIMMERMAn Eric (2004), Rules of Play: Game Design Fundamentals, Cambridge, MIT Press. 
sснотт Gareth Richard (2011). « The production of machinima: A dialogue between ethnography, culture and space », International Journal of Business, Humanities and Technology, 1 (1), pp. 113-121.

STEIN Louisa Ellen \& BUSSE Kristina (2009). « Limit Play: Fan Authorship between Source Text, Intertext, and Context », Popular Communication, 7 (4), pp. 192-207.

TRICLOT Mathieu (2011). Philosophie des jeux vidéo, Paris, La Découverte.

VIAL Stéphane (2012). « Pour introduire le "playsir" : jouabilité psychique et ludogénéité numérique ", Communication présentée au colloque Jeu et jouabilité à l'ère numérique, Paris, 8 décembre 2012.

WENDLING Thierry (2002). Ethnologie des joueurs d'échecs, Paris, Presses Universitaires de France.

\section{ANNEXES}

\section{Sources primaires citées}

\section{Fanfictions}

- «APAGCPMDEO », sur Fanfiction.net. URL : https://www.fanfiction.net/forum/ APAGCPMDEO/56737/

- «Ariani Lee », sur Fanfiction.net. URL: https://www.fanfiction.net/u/1331270/ Ariani-Lee

- "Axel et Roxas dans cent thèmes, chapitre 1 ", sur Fanfiction.net. URL : https:// www.fanfiction.net/s/5360298/1/Axel-et-Roxas-dans-cent-th \%C3 \%A8mes

- "Axel et Roxas dans cent thèmes, chapitre 2 ", sur Fanfiction.net. URL : https:// www.fanfiction.net/s/5360298/2/Axel-et-Roxas-dans-cent-th \%C3 \%A8mes

- «Crushcrushcrush, chapitre 15 », sur Fanfiction.net. URL: https:// www.fanfiction.net/s/7107693/15/Crushcrushcrush

- «Crushcrushcrush, chapitre 43 », sur Fanfiction.net. URL: https:// www.fanfiction.net/s/7107693/43/Crushcrushcrush

- «FFN Research », sur Fan Fiction Statistics. URL : http://ffnresearch.blogspot.be/

- «FIC », sur Fanfiction.net. URL : https://www.fanfiction.net/forum/FIC/77278/

- «I've lost my heart in winter, chapitre 1 », sur Fanfiction.net. URL: https:// www.fanfiction.net/s/5609560/1/I-ve-lost-my-heart-in-winter

- «Kokoro ga nai, chapitre 6 », sur Fanfiction.net. URL : https://www.fanfiction.net/ s/5874607/6/Kokoro-ga-nai

- «Les Bannis ont droit d'amour, chapitre 3 », sur Fanfiction.net. URL : https:// www.fanfiction.net/s/4975923/3/Les-Bannis-ont-droit-d-amour

- «Les Bannis ont droit d'amour, chapitre 29 ", sur Fanfiction.net. URL: https:// www.fanfiction.net/s/4975923/29/Les-Bannis-ont-droit-d-amour

- «Les Chroniques de l'Héritier Déchu», sur Fanfiction.net. URL: https:// www.fanfiction.net/s/4013327/1/Les-Chroniques-de-l-H \%C3 \%A9ritier-D \%C3 \% A9chu

- «Les OC /Original Character ou Personnage Original/", sur Fanfiction.net. URL : https://www.fanfiction.net/topic/77279/35495773/1/Les-OC-Original-Character-ouPersonnage-Original 
- «MAUVE, première partie: À cœur fendre, chapitre 1 », sur Fanfiction.net. URL: https://www.fanfiction.net/s/6422643/1/MAUVE-premi \%C3 \%A8re-partie-A-coeurfendre

- «My Sweet Dangerous Cat, chapitre 1», sur Fanfiction.net. URL: https:// www.fanfiction.net/s/4751757/1/My-Sweet-Dangerous-Cat

- «Parlons fanfiction », sur Fanfiction.net. URL: https://www.fanfiction.net/forum/ Parlons-fanfiction/77279/

- «Puisque la folie te guette, chapitre 2 ", sur Fanfiction.net. URL: https:// www.fanfiction.net/s/5807613/2/Puisque-la-folie-te-guette

- «Puisque la folie te guette, chapitre 30 », sur Fanfiction.net. URL: https:// www.fanfiction.net/s/5807613/30/Puisque-la-folie-te-guette

- «Requests for Constructive Criticism and Tips for Writing/Getting Reviews », sur Fanfiction.net. URL : https://www.fanfiction.net/topic/2872/8487857/1/Requests-forConstructive-Criticism-and-Tips-for-Writing-Getting-Reviews

- «Valse de la Lune", sur Fanfiction.net. URL: https://www.fanfiction.net/ s/3690783/1/Valse-de-la-Lune

Machinimas

- «[Machinima] Le Mur (Spécial 700 Abonnés)», sur YouTube. URL: https:// www.youtube.com/watch?

$\mathrm{v}=$ NyH4IQal6a8\&list $=$ UUfMaEkC5rPoX696mpJDI7gg\&index $=4$

- « [MachinimaHD Halo 4] Poursuit », sur YouTube. URL : https://www.youtube.com/ watch ?v =E6dJcVwQ2Ik\&index =18\&list =UUfMaEkC5rPoX696mpJDI7gg

- « About », sur This Spartan Life. URL : http://www.thisspartanlife.com/sample-page/

- «Arby and The Chief : Season 1 », sur YouTube. URL : https://www.youtube.com/ watch ?v =kb8rdc-00pQ\&index =1\&list =PL104A38C965EDA0FC

- «Arby ' $n$ ' the Chief, Conflict - Part 1 » $(08: 28)$, sur YouTube. URL: https:// www.youtube.com/watch ?v =GsoXlpnPF90\&index =11\&list =PL104A38C965EDA0FC

- "Common Sense Cooking with Carl the Cook», sur Internet Archive. URL : https:// archive.org/details/common_sense_cooking

- «Company History », sur Rooster Teeth. URL: http://roosterteeth.com/about/ history.php

- «Coverage », sur Rooster Teeth. URL : http://roosterteeth.com/about/coverage.php)

- «Des vacances qui commencent bien!", sur YouTube. URL: https:// www.youtube.com/watch?

$\mathrm{v}=$ =FGf0fG81ip8\&index =8\&list =UU_ANJ8SEtUE8Y33x2LCXNwA

- «DigitalPh33r's Guide to Making Halo 3 Machinima », sur YouTube. URL : https:// www.youtube.com/watch ?v =Eu3qxeZmBrk

- «Les petites bêtes", sur YouTube. URL: https://www.youtube.com/watch? $\mathrm{v}=$ HkkTln-AWsQ\&list =UUVuCJG5RPwnVXoqbwGSBoag

- «Reconstruction Trailer», sur Rooster Teeth. URL: http://roosterteeth.com/ archive/?id=277\&v=more\&s=6

- «This Spartan Life », sur Chaos Control Digizine. URL : http://www.chaoscontrol.com/ this-spartan-life/

- «Zardoz Partie 1 - Le film d'animation », sur Kingrabbit.fr. URL: http:// kingrabbit.over-blog.com/article-zardoz-partie-1-le-film-d-

animation-93202605.html 
- Delaney Kevin J. (9 avril 2004), « When Art Imitates Videogames, You Have "Red vs. Blue" », The Wall Street Journal, Dow Jones \& Company. [Disponible en ligne sur The Wall Street Journal. URL : http://online.wsj.com/articles/SB108145721789778243]

- Fitzgerald Courtney (2004), «Almost famous : Red vs. Blue's machinima stars », sur The Austin Chronicle. URL: http://www.austinchronicle.com/ screens/2004-03-05/200235/

- Moltenbrey Karen (2005), « Out of Character », Computer Graphics World, vol. 28, n 11 [Disponible en ligne sur Computer Graphics World. URL: http://www.cgw.com/ Publications/CGW/2005/Volume-28-Issue-11-November-2005-/Out-of-

Character.aspx]

Speedrun et tool-assisted speedrun

- «Guidelines », sur TASVideos. URL : http://tasvideos.org/Guidelines.html

- «Are Tool-Assisted Runs Conceptual Art?», sur TASVideos. URL: http:// tasvideos.org/forum/viewtopic.php?t=14435

- "As Fast as Impossible: 10 Insanely Thrilling Tool-Assisted Speedruns », sur Crispy Gamer. URL: http://www.crispygamer.com/features/2009-04-20/as-fast-asimpossible-10-insanely-thrilling-tool-assisted-speedruns.aspx

- «Debate: allowed or not?», sur TASVideos. URL: http://tasvideos.org/forum/ viewtopic.php?

$\mathrm{t}=14051$ \&postdays $=0$ \&postorder=asc\&start=0\&sid=2e04076f5242473eaf0abc8aac8b61a6

- «Dragon Warrior », sur Speed Demos Archive. URL : http://speeddemosarchive.com/ DragonWarrior.html

- "Frequently Asked Questions", sur Speed Demos Archive. URL: http:// www.speeddemosarchive.com/lang/faq_en.html

- «Interview of adelikat for GamersGlobal », sur TASVideos. URL : http://tasvideos.org/ Interviews/Adelikat/GamersGlobal2010.html

- «NES Gradius in 10:52.35 by adelikat », sur TASVideos. URL : http://tasvideos.org/ Game/nes-gradius.html

- «SDA Knowledge Base», sur Speed Demos Archive. URL: https:// $\mathrm{kb}$.speeddemosarchive.com/Main_Page

- «Spelunker», sur Speed Demos Archive. URL: http://speeddemosarchive.com/ Spelunker.html

- «Submission \#2990 : Ryuto's DS Brain Age in 06 :33.66 », sur TASVideos. URL : http:// tasvideos.org/2990S.html

- «Submission \#3039: Kriole's GBA Castlevania: Aria of Sorrow "100\% souls" in $24: 56.1 »$, sur TASVideos. URL : http://tasvideos.org/3039S.html

- «Submission \#3426: Mukki's GBA Sonic Advance 2 in 18 :01.78», sur TASVideos. URL : http://tasvideos.org/3426S.html

- «Submission \#3557: Aglar's Genesis Sonic the Hedgehog 2 in 17 :51.6», sur TASVideos . URL : http://tasvideos.org/3557S.html

- «Super Mario Bros », sur Speed Demos Archive. URL : http://speeddemosarchive.com/ Mario1.html

- "TAS HD : Genesis Sonic the Hedgehog 2 in $17: 51.6$ by Aglar (2 screens)», sur YouTube. URL : https://www.youtube.com/watch ?v =cPN9fv8CAgw

- «Welcome To TAS Videos", sur TASVideos. URL: http://tasvideos.org/ WelcomeToTASVideos.html 
- Teti John, « As Fast as Impossible : 10 Insanely Thrilling Tool-Assisted Speedruns », sur Crispy Gamer. URL : http://www.crispygamer.com/features/2009-04-20/as-fastas-impossible-10-insanely-thrilling-tool-assisted-speedruns.aspx

Modding

- «Alternate Start - Live Another Life», sur Skyrim Nexus mods and community. URL : http://www.nexusmods.com/skyrim/mods/9557/?

- «Cooking Mama, The Unauthorized PETA Edition : Mama Kills Animals », sur PETA People for the Ethical Treatment of Animals. URL: http://features.peta.org/ CookingMama/

- «Frostfall - Hypothermia Camping Survival », sur Skyrim Nexus mods and community. URL : http://www.nexusmods.com/skyrim/mods/11163/?

- «Nexus Wiki ", sur Nexus mods and community. URL: http://wiki.tesnexus.com/ index.php/Main_Page

- «RaceMenu», sur Skyrim Nexus mods and community. URL: http:// www.nexusmods.com/skyrim/mods/29624/?

- «SkyUI ", sur Skyrim Nexus mods and community. URL : http://www.nexusmods.com/ skyrim/mods/3863/?

- «Unofficial Skyrim Patch», sur Skyrim Nexus mods and community. URL: http:// www.nexusmods.com/skyrim/mods/19/?

Autres

- «Twitch plays Pokémon» sur Twitch. URL: http://www.twitch.tv/ twitchplayspokemon

- Ludographie ${ }^{\mathbf{8 8}}$

- Adventure (William Crowther et Don Woods, 1977)

- Castlevania : Aria of Sorrow (Konami, 2003)

- City of Heroes (Cryptic Studios, NCsoft, 2004)

- Cooking Mama (Office Create, Taito, 2006)

- Counter-Strike (Valve Corporation, Microsoft Studios, 2000)

- DOOM (Id Software, 1993)

- Dragon Warrior (Chunsoft, Enix/Nintendo, 1986)

- Final Fantasy VII (Square, Sony Computer Entertainment, 1997)

- Gradius (Konami, 1986)

- Grand Theft Auto (Rockstar Games, Take 2 Interactive, 1997)

- Half-Life (Valve Corporation, Sierra On-Line, 1998)

- Halo 2 (Bungie Software, Microsoft Studios, 2004)

- Halo : Combat Evolved (Bungie Software, Microsoft Studios, 2001)

- Kingdom Hearts (Square, Sony Computer Entertainment, 2002)

- Les Sims (Maxis, Electronic Arts, 2000)

- Little Big Planet (Media Molecule, Sony Computer Entertainment, 2008)

- Minecraft (4J Studios, Mojang, 2011)

- Pokémon Rouge et Bleu (Game Freak, Nintendo, 1996)

- Programme d'entraînement cérébral du Dr Kawashima: Quel âge a votre cerveau? (Nintendo, 2005)

- Sonic Advance 2 (Sega, 2002)

- Sonic the Hedgehog 2 (Sega, 1992)

- Spacewar (Steve Russell, John Graetz, Wayne Wiitanen et al., 1962)

- Spelunker (MicroGraphicImage, 1983) 
- Super Mario Bros (Nintendo, 1985)

- Super Mario 64 (Nintendo, 1996)

- Super Metroid (Nintendo, 1994)

- The Elder Scrolls V : Skyrim (Bethesda Softworks, Ubisoft, 2011)

- TrackMania (Nadeo, Focus Home Interactive, 2003)

- World of Warcraft (Blizzard Entertainment, 2005)

\section{Corpus}

Fanfictions

- « Kokoro ga nai », sur Fanfiction.net. URL : https://www.fanfiction.net/s/5874607/1/ Kokoro-ga-nai

- «Puisque la folie te guette », sur Fanfiction.net. URL : https://www.fanfiction.net/ s/5807613/1/Puisque-la-folie-te-guette

- «Esprit du Feu », sur Fanfiction.net. URL : https://www.fanfiction.net/s/5491043/1/ Esprit-du-Feu

- "Axel et Roxas dans cent thèmes ", sur Fanfiction.net. URL: https:// www.fanfiction.net/s/5360298/1/Axel-et-Roxas-dans-cent-th \%C3 \%A8mes

- «Les Bannis ont droit d'amour», sur Fanfiction.net. URL: https:// www.fanfiction.net/s/4975923/1/Les-Bannis-ont-droit-d-amour

- «MAUVE, première partie : À cœur fendre ", sur Fanfiction.net. URL: https:// www.fanfiction.net/s/6422643/1/MAUVE-premi \%C3 \%A8re-partie-A-coeur-fendre

- «My Sweet Dangerous Cat », sur Fanfiction.net. URL : https://www.fanfiction.net/ s/4751757/1/My-Sweet-Dangerous-Cat

- «Crushcrushcrush», sur Fanfiction.net. URL : https://www.fanfiction.net/ s/7107693/1/Crushcrushcrush

- «Double Je», sur Fanfiction.net. URL : https://www.fanfiction.net/s/3765742/1/ Double-Je

- «Liste de choses que Demyx ne peut plus faire », sur Fanfiction.net. URL : https:// www.fanfiction.net/s/5448953/1/Liste-de-choses-que-Demyx-ne-peut-plus-faire

- «I've lost my heart in winter », sur Fanfiction.net. URL : https://www.fanfiction.net/ s/5609560/1/I-ve-lost-my-heart-in-winter

- «Les Chroniques de l'Héritier Déchu», sur Fanfiction.net. URL: https:// www.fanfiction.net/s/4013327/1/Les-Chroniques-de-l-H \%C3 \%A9ritier-D \%C3 \% A9chu

- «Coup de Foudre à Disneyland», sur Fanfiction.net. URL: https:// www.fanfiction.net/s/9422009/1/Coup-de-Foudre- \%C3 \%A0-Disneyland

- «Mémoires de 24 chocolats ", sur Fanfiction.net. URL : https://www.fanfiction.net/ s/4689011/1/M \%C3 \%A9moires-de-24-chocolats

- "Valse de la Lune", sur Fanfiction.net. URL: https://www.fanfiction.net/ s/3690783/1/Valse-de-la-Lune

- «Seconde Chance", sur Fanfiction.net. URL: https://www.fanfiction.net/ s/4575438/1/Seconde-Chance

- «Quand on est en colocation », sur Fanfiction.net. URL : https://www.fanfiction.net/ s/4557947/1/Quand-on-est-en-colocation

- «Hors de Portée », sur Fanfiction.net. URL : https://www.fanfiction.net/s/5050689/1/ Hors-de-Port \%C3 \%A9e

- «Un nouveau départ», sur Fanfiction.net. URL: https://www.fanfiction.net/ s/3504762/1/Un-nouveau-d \%C3 \%A9part 
- «A song for a smile», sur Fanfiction.net. URL: https://www.fanfiction.net/ s/6586893/1/A-song-for-a-smile

- «Quand on va à la neige », sur Fanfiction.net. URL: https://www.fanfiction.net/ s/3715675/1/Quand-on-va- \%C3 \%A0-la-neige

- «Les vacances de l'organisation XIII", sur Fanfiction.net. URL: https:// www.fanfiction.net/s/4241921/1/Les-vacances-de-l-organisation-XIII

- «Pour l'Amour de l'Air», sur Fanfiction.net. URL: https://www.fanfiction.net/ s/5862672/1/Pour-l-Amour-de-l-Air

- «Croire au père Noël », sur Fanfiction.net. URL: https://www.fanfiction.net/ s/4739400/1/Croire-au-p \%C3 \%A8re-No \%C3 \%ABl

- «Pour manger des glaces en Enfer », sur Fanfiction.net. URL: https:// www.fanfiction.net/s/5897344/1/Pour-manger-des-glaces-en-Enfer

- «Les Âmes Damnées ", sur Fanfiction.net. URL: https://www.fanfiction.net/ s/5874498/1/Les- \%C3 \%82mes-Damn \%C3 \%A9es

- «Un triangle parfait», sur Fanfiction.net. URL: https://www.fanfiction.net/ s/6430155/1/Un-triangle-parfait

- «Tout un art», sur Fanfiction.net. URL : https://www.fanfiction.net/s/3891567/1/ Tout-un-art

- «Lorsqu'on veut se plaire", sur Fanfiction.net. URL : https://www.fanfiction.net/ s/5687636/1/Lorsqu-on-veut-se-plaire

- «Les Enfants du Chaos », sur Fanfiction.net. URL: https://www.fanfiction.net/ s/4161511/1/Les-Enfants-du-Chaos

Machinimas

L'analyse a porté sur l'ensemble des vidéos basées sur la licence Halo et produites par les machinimakers (ou groupes de machinimakers) suivants :

- BangBang TV, sur YouTube. URL : https://www.youtube.com/user/bigredlink6

- Bong + Dern Productions, sur This Spartan Life. URL: http:// www.thisspartanlife.com/about.php

- $\mathrm{CFH}$ Fan, sur YouTube. URL: https://www.youtube.com/channel/ UCVuCJG5RPwnVXoqbwGSBoag

- DefendTheHouse, sur YouTube. URL: https://www.youtube.com/user/ defendthehouse

- Digitalph33r Productions, sur Digitalph33r Productions. URL: http:// digitalph33r.blogspot.be/

- Djoey154, sur Halo.Bungie.org. URL : http://halo.bungie.org/misc/spicymirrors.html

- Edgeworks, sur Edgeworks Entertainment. URL : http://www.edgeworksent.com/

- Fire Team Charlie, sur Xboxottawa.ca. URL : http://www.xboxottawa.ca/

- FulRoro, sur YouTube. URL : https://www.youtube.com/user/FulRoro

- GeneticSpartan, sur YouTube. URL : https://www.youtube.com/geneticspartan\#play/ uploads/3/eadPgHFZrLw

- Monty Oum, sur Montyoum.net. URL : http://montyoum.net/

- Numérimax Studio, sur YouTube. URL: https://www.youtube.com/user/ FunHouseProd/about

- Opportunist, sur YouTube. URL : https://www.youtube.com/user/0pportunist

- Orbital Studios, sur Orbital Forum. URL : http://orbitalstudios.highforum.net/t501dailymotion-orbital-pack 
- Rooster Teeth Productions, sur Rooster Teeth. URL: http://roosterteeth.com/ home.php

- StaffG \& Jefferson, sur YouTube. URL : https://www.youtube.com/user/StaffG

- Team CRéA, sur Dailymotion. URL: http://www.dailymotion.com/playlist/ xs81v_Co0oX_halo-creation/1\#video=xcubd3

- TGO GMBH, sur TGO GMBH. URL : http://tgo-gmbh.net/fr/

- Vandya, sur YouTube. URL : https://www.youtube.com/watch ?v =tRWxmCeBq7w

- Yupjo, sur Dailymotion. URL : http://www.dailymotion.com/yupjo\#video=x46ftr

- Zukan, sur YouTube. URL : https://www.youtube.com/user/Zukan/videos

- Speedrun et tool-assisted speedrun

- «E.T. The Extra-Terrestrial», sur Speed Demos Archive. URL: http:// speeddemosarchive.com/ET.html

- «Spelunker», sur Speed Demos Archive. URL: http://speeddemosarchive.com/ Spelunker.html

- «Donkey Kong Jr. », sur Speed Demos Archive. URL : http://speeddemosarchive.com/ DonkeyKongJr.html

- «Pitfall II : Lost Caverns (0:05:02 by Tom Batchelor)», sur Speed Demos Archive. URL : http://speeddemosarchive.com/Pitfall2.html

- «Pitfall II : Lost Caverns (100 \%: 0 :09:47 by Chris Knight) », sur Speed Demos Archive. URL : http://speeddemosarchive.com/Pitfall2.html

- «Kung Fu», sur Speed Demos Archive. URL: http://speeddemosarchive.com/ KungFu.html

- «Excitebike», sur Speed Demos Archive. URL: http://speeddemosarchive.com/ Excitebike.html

- «Super Mario Bros. (0:04:58 by Andrew Gardikis)», sur Speed Demos Archive. URL : http://speeddemosarchive.com/Mario1.html

- «Super Mario Bros. (100 \%: $0: 19: 40$ by Andrew Gardikis)", sur Speed Demos Archive. URL : http://speeddemosarchive.com/Mario1.html

- «Ikari Warriors 2: Victory Road», sur Speed Demos Archive. URL: http:// speeddemosarchive.com/IkariWarriors2.html

- «Donkey Kong», sur Speed Demos Archive. URL : http://speeddemosarchive.com/ DonkeyKong.html

- «Super Mario Bros. : The Lost Levels (Wii/Famicom as Mario : $0: 08: 13$ by Andrew Gardikis)", sur Speed Demos Archive. URL: http://speeddemosarchive.com/ MarioLostLevels.html

- "Super Mario Bros.: The Lost Levels (SNES Lost Levels Single-segment as Mario $0: 18: 05$ by Tom Votava)», sur Speed Demos Archive. URL: http:// speeddemosarchive.com/MarioLostLevels.html

- «Super Mario Bros.: The Lost Levels (SNES Lost Levels Single-segment as Luigi 0:16:07 by 奥村 亮)», sur Speed Demos Archive. URL: http:// speeddemosarchive.com/MarioLostLevels.html

- "Super Mario Bros. : The Lost Levels (SNES Lost Levels Single-segment $100 \%$ as Luigi $0: 37: 58$ by 奥村 亮)», sur Speed Demos Archive. URL: http:// speeddemosarchive.com/MarioLostLevels.html

- «Metroid (One-boss run $0: 15$ :36 by Chris Knight)», sur Speed Demos Archive. URL : http://speeddemosarchive.com/Metroid.html

- «Metroid (Up+a warping $0: 15: 35$ by 'jprophet22')», sur Speed Demos Archive. URL : http://speeddemosarchive.com/Metroid.html 
- «Metroid (Both bosses beaten $0: 24$ :06 by Chris Knight)», sur Speed Demos Archive. URL : http://speeddemosarchive.com/Metroid.html

- «Metroid (100 \% $0: 52: 23$ by Damien Moody)», sur Speed Demos Archive. URL : http:// speeddemosarchive.com/Metroid.html

- "Alex Kidd in Miracle World», sur Speed Demos Archive. URL: http:// speeddemosarchive.com/AlexKiddMiracleWorld.html

- "Ghosts 'n Goblins », sur Speed Demos Archive. URL : http://speeddemosarchive.com/ GhostsnGoblins.html

- « King Kong 2 : Ikari No Megaton Punch (0:03:34 by Elijah Miller)», sur Speed Demos Archive. URL : http://speeddemosarchive.com/KingKong2.html

- «King Kong 2 : Ikari No Megaton Punch (All bosses : $0: 10: 08$ by Elijah Miller)», sur Speed Demos Archive. URL : http://speeddemosarchive.com/KingKong2.html

- «Mighty Bomb Jack (0:06:57 by Dag Cato)», sur Speed Demos Archive. URL : http:// speeddemosarchive.com/MightyBombJack.html

- «Mighty Bomb Jack (Best Ending with deaths : $0: 12: 06$ by Dag Cato)», sur Speed Demos Archive. URL : http://speeddemosarchive.com/MightyBombJack.html

- "Skull and Crossbones», sur Speed Demos Archive. URL: http:// speeddemosarchive.com/SkullAndCrossbones.html

- «Zillion», sur Speed Demos Archive. URL: http://speeddemosarchive.com/ Zillion.html

- "Trojan", sur Speed Demos Archive. URL: http://speeddemosarchive.com/ Trojan.html

- «Pro Wrestling», sur Speed Demos Archive. URL : http://speeddemosarchive.com/ ProWrestling.html

- «Rush 'n Attack», sur Speed Demos Archive. URL : http://speeddemosarchive.com/ RushnAttack.html

- «Castlevania», sur Speed Demos Archive. URL: http://speeddemosarchive.com/ Castlevania.html

- «Dragon Warrior », sur Speed Demos Archive. URL : http://speeddemosarchive.com/ DragonWarrior.html

- «Athena», sur Speed Demos Archive. URL: http://speeddemosarchive.com/ Athena.html

- «Kid Icarus", sur Speed Demos Archive. URL: http://speeddemosarchive.com/ KidIcarus.html

- «Metal Gear (Single-segment with deaths $0: 27: 57$ by Marko Vanhanen) », sur Speed Demos Archive. URL : http://speeddemosarchive.com/MetalGear.html

- «Metal Gear (Single-segment 0:31:55 by Marko Vanhanen)», sur Speed Demos Archive. URL : http://speeddemosarchive.com/MetalGear.html

- «Submission \#4364: £e Nécroyeur's Arcade Magician Lord in 07:17.73», sur TASVideos. URL : http://tasvideos.org/4364S.html

- «Submission \#3685: SDR's Arcade Marvel vs. Capcom "Playaround" in 25 :37.7 », sur TASVideos. URL : http://tasvideos.org/3685S.html

- "Submission \#3125: x2poet's Arcade Metal Slug X - Super Vehicle-001 in 18:32.2», sur TASVideos. URL : http://tasvideos.org/3125S.html

- "Submission \#2821: adelikat \& DarkKobold's Arcade The Simpsons: The Arcade Game "4-player" in $13: 37.73$ ", sur TASVideos. URL : http://tasvideos.org/2821S.html

- «Submission \#4095 : slamo's DOS Jetpack in 32 :22.56», sur TASVideos. URL : http:// tasvideos.org/4095S.html 
- « Submission \#2990 : Ryuto's DS Brain Age in 06 :33.66», sur TASVideos. URL : http:// tasvideos.org/2990S.html

- «Submission \#3590 : VanillaCoke's DS Sonic Colors in 26 :37.8 », sur TASVideos. URL: http://tasvideos.org/3590S.html

- « Submission \#4016: Chef Stef \& Kiwisauce's DS Super Scribblenauts in 1 :01:52.63 », sur TASVideos. URL : http://tasvideos.org/4016S.html

- «Submission \#3039: Kriole's GBA Castlevania: Aria of Sorrow "100\% souls" in 24 :56.1», sur TASVideos. URL : http://tasvideos.org/3039S.html

- "Submission \#4069: Dragonfangs's GBA Metroid Zero Mission "100\%" in $1: 00: 46.28 »$, sur TASVideos. URL : http://tasvideos.org/4069S.html

- «Submission \#2172 : Cardboard's GBA Ninja Five-O in 16 :35.62 », sur TASVideos. URL : http://tasvideos.org/2172S.html

- «Submission \#4390: GoddessMaria, Grincevent \& VanillaCoke's GBA Sonic Advance "Amy" in 15 :25.8 ", sur TASVideos. URL : http://tasvideos.org/4390S.html

- «Submission \#3426: Mukki's GBA Sonic Advance 2 in 18 :01.78», sur TASVideos. URL : http://tasvideos.org/3426S.html

- «Submission \#3002 : andymac's GBA Wario Land 4 in 40 :04.88 », sur TASVideos. URL: http://tasvideos.org/3002S.html

- «Submission \#3894 : FractalFusion's GBC Pokémon Yellow "Executes Arbitrary Code" in 03 :14.15 », sur TASVideos. URL : http://tasvideos.org/3894S.html

- «Submission \#2565: Swordless Link's GBC The Legend of Zelda: Link's Awakening DX in $1: 00: 02.68 »$, sur TASVideos. URL : http://tasvideos.org/2565S.html

- «Submission \#3579: bobmario511's GB The Legend of Zelda: Link's Awakening "Playaround" in $40: 22.78$ ", sur TASVideos. URL : http://tasvideos.org/3579S.html

- «Submission \#2923 : Greenalink's GB Wario Land - Super Mario Land 3 in $21: 49.15$ », sur TASVideos. URL : http://tasvideos.org/2923S.html

- «Submission \#4038 : gamerfreak5665 \& aleckermit's GC 007 : Nightfire in 39 :55.45 », sur TASVideos. URL : http://tasvideos.org/4038S.html

- «Submission \#4078: THC98's GC Sonic Adventure DX: Director's Cut "Sonic" in $30: 12.02 »$, sur TASVideos. URL : http://tasvideos.org/4078S.html

- «Submission \#4116: numerics's GC Super Smash Bros. Melee "Adventure Mode" in $10: 23.48 »$, sur TASVideos. URL : http://tasvideos.org/4116S.html

- «Submission \#2604: Mukki's Genesis Golden Axe in 07 :01.95 », sur TASVideos. URL : http://tasvideos.org/2604S.html

- «Submission \#1645: arkiandruski's Genesis Gunstar Heroes in 35 :28.92 », sur TASVideos. URL : http://tasvideos.org/1645S.html

- «Submission \#2877 : Nitsuja \& Upthorn \& marzojr's Genesis Sonic 3 \& Knuckles in 29 :51.2 », sur TASVideos. URL : http://tasvideos.org/2877S.html

- « Submission \#3557 : Aglar's Genesis Sonic the Hedgehog 2 in $17: 51.6$ », sur TASVideos . URL : http://tasvideos.org/3557S.html

Modding

- «SkyUI", sur Skyrim Nexus - mods and community. URL: http:// www.nexusmods.com/skyrim/mods/3863/?

- "A Quality World Map - With Roads ", sur Skyrim Nexus - mods and community. URL : http://www.nexusmods.com/skyrim/mods/4929/?

- "Immersive Armors", sur Skyrim Nexus - mods and community. URL: http:// www.nexusmods.com/skyrim/mods/19733/? 
- «ApachiiSkyHair", sur Skyrim Nexus - mods and community. URL: http:// www.nexusmods.com/skyrim/mods/10168/?

- «Unofficial Skyrim Patch", sur Skyrim Nexus - mods and community. URL : http:// www.nexusmods.com/skyrim/mods/19/?

- "Climates Of Tamriel - Weather - Lighting - Audio", sur Skyrim Nexus - mods and community. URL : http://www.nexusmods.com/skyrim/mods/17802/ ?

- «Enhanced Blood Textures ", sur Skyrim Nexus - mods and community. URL : http:// www.nexusmods.com/skyrim/mods/60/?

- «Unofficial Dawnguard Patch », sur Skyrim Nexus - mods and community. URL : http:// www.nexusmods.com/skyrim/mods/23491/?

- «Skyrim HD - 2K Textures », sur Skyrim Nexus - mods and community. URL : http:// www.nexusmods.com/skyrim/mods/607/?

- «Unofficial Dragonborn Patch», sur Skyrim Nexus - mods and community. URL: http://www.nexusmods.com/skyrim/mods/31083/?

- «Immersive Weapons", sur Skyrim Nexus - mods and community. URL: http:// www.nexusmods.com/skyrim/mods/27644/?

- «Unofficial Hearthfire Patch », sur Skyrim Nexus - mods and community. URL : http:// www.nexusmods.com/skyrim/mods/25127/?

- "Cloaks of Skyrim», sur Skyrim Nexus - mods and community. URL: http:// www.nexusmods.com/skyrim/mods/12092/?

- «RaceMenu", sur Skyrim Nexus - mods and community. URL: http:// www.nexusmods.com/skyrim/mods/29624/?

- «Static Mesh Improvement Mod - SMIM », sur Skyrim Nexus - mods and community. URL : http://www.nexusmods.com/skyrim/mods/8655/?

- «Fores New Idles in Skyrim - FNIS », sur Skyrim Nexus - mods and community. URL : http://www.nexusmods.com/skyrim/mods/11811/?

- «Alternate Start - Live Another Life», sur Skyrim Nexus - mods and community. URL : http://www.nexusmods.com/skyrim/mods/9557/?

- «WATER - Water And Terrain Enhancement Redux », sur Skyrim Nexus - mods and community. URL : http://www.nexusmods.com/skyrim/mods/13268/ ?

- «UFO - Ultimate Follower Overhaul », sur Skyrim Nexus - mods and community. URL : http://www.nexusmods.com/skyrim/mods/14037/?

- «The Dance of Death - A Killmove Mod», sur Skyrim Nexus - mods and community. URL : http://www.nexusmods.com/skyrim/mods/10906/?

- «Skyrim Flora Overhaul», sur Skyrim Nexus - mods and community. URL : http:// www.nexusmods.com/skyrim/mods/141/?

- «Wet and Cold», sur Skyrim Nexus - mods and community. URL: http:// www.nexusmods.com/skyrim/mods/27563/?

- «Frostfall - Hypothermia Camping Survival », sur Skyrim Nexus - mods and community. URL : http://www.nexusmods.com/skyrim/mods/11163/?

- «Convenient Horses", sur Skyrim Nexus - mods and community. URL: http:// www.nexusmods.com/skyrim/mods/14950/?

- «Realistic Ragdolls and Force ", sur Skyrim Nexus - mods and community. URL : http:// www.nexusmods.com/skyrim/mods/601/?

- «Enhanced Lights and FX», sur Skyrim Nexus - mods and community. URL : http:// www.nexusmods.com/skyrim/mods/27043/?

- «T3nd0s Skyrim Redone", sur Skyrim Nexus - mods and community. URL : http:// www.nexusmods.com/skyrim/mods/9286/? 
- «Immersive HUD - iHUD», sur Skyrim Nexus - mods and community. URL : http:// www.nexusmods.com/skyrim/mods/3222/?

- «Falskaar", sur Skyrim Nexus - mods and community. URL: http:// www.nexusmods.com/skyrim/mods/37994/?

- «RealVision ENB», sur Skyrim Nexus - mods and community. URL: http:// www.nexusmods.com/skyrim/mods/30936/?

- « Realistic Lighting Overhaul », sur Skyrim Nexus - mods and community. URL : http:// www.nexusmods.com/skyrim/mods/30450/?

- «Interesting NPCs», sur Skyrim Nexus - mods and community. URL: http:// www.nexusmods.com/skyrim/mods/8429/?

- «Unofficial High Resolution Patch », sur Skyrim Nexus - mods and community. URL : http://www.nexusmods.com/skyrim/mods/31255/?

- «Skyrim -Community- Uncapper», sur Skyrim Nexus - mods and community. URL : http://www.nexusmods.com/skyrim/mods/1175/?

- "The Eyes Of Beauty", sur Skyrim Nexus - mods and community. URL: http:// www.nexusmods.com/skyrim/mods/13722/?

- "JaySuS Swords", sur Skyrim Nexus - mods and community. URL: http:// www.nexusmods.com/skyrim/mods/1002/?

- «TES5Edit», sur Skyrim Nexus - mods and community. URL: http:// www.nexusmods.com/skyrim/mods/25859/?

- «Better Females by Bella », sur Skyrim Nexus - mods and community. URL : http:// www.nexusmods.com/skyrim/mods/2812/?

- «Deadly Dragons", sur Skyrim Nexus - mods and community. URL: http:// www.nexusmods.com/skyrim/mods/3829/?

- "aMidianBorn Book of Silence", sur Skyrim Nexus - mods and community. URL: http://www.nexusmods.com/skyrim/mods/24909/?

- "Auto Unequip Ammo », sur Skyrim Nexus - mods and community. URL: http:// www.nexusmods.com/skyrim/mods/10753/?

- «Winter Is Coming - Cloaks », sur Skyrim Nexus - mods and community. URL : http:// www.nexusmods.com/skyrim/mods/13486/?

- «Run For Your Lives", sur Skyrim Nexus - mods and community. URL: http:// www.nexusmods.com/skyrim/mods/23906/?

- "Bandolier - Bags and Pouches", sur Skyrim Nexus - mods and community. URL: http://www.nexusmods.com/skyrim/mods/16438/?

- «Glowing Ore Veins $300 »$, sur Skyrim Nexus - mods and community. URL : http:// www.nexusmods.com/skyrim/mods/193/?

- «Amazing Follower Tweaks », sur Skyrim Nexus - mods and community. URL : http:// www.nexusmods.com/skyrim/mods/15524/?

- «Mod Organizer», sur Skyrim Nexus - mods and community. URL: http:// www.nexusmods.com/skyrim/mods/1334/?

- «Guard Dialogue Overhaul», sur Skyrim Nexus - mods and community. URL : http:// www.nexusmods.com/skyrim/mods/23390/?

- "Apocalypse - Magic of Skyrim», sur Skyrim Nexus - mods and community. URL: http://www.nexusmods.com/skyrim/mods/16225/?

- «Enhanced Character Edit », sur Skyrim Nexus - mods and community. URL : http:// www.nexusmods.com/skyrim/mods/12951/? 


\section{NOTES}

1. La mise à disposition, à partir de 1997, du code source du jeu DOOM (1993) par ses concepteurs - qui a permis aux utilisateurs de créer et de diffuser leurs propres niveaux de jeu, convertissant ainsi ce public en un réservoir de producteurs bénévoles de contenu - en est une illustration.

2. Les amateurs n'ont, par exemple, pas attendu Internet pour s'échanger des productions littéraires ou graphiques inspirées de leurs œuvres favorites, à l'image du contenu des magazines de fans (ou fanzines), autrefois sous format papier. L'évolution de ces pratiques a notamment été décrite par François (2008) pour les fanfictions et Jenkins (1992 et 2006) pour plusieurs pratiques de fans; on peut également, selon Triclot, faire remonter la pratique du «modding " (voir cidessous) aux premiers jeux vidéo, nés dans l'écosystème universitaire (Spacewar, Adventure et leurs nombreuses imitations, entre autres), qui étaient « destinés à être joués, mais aussi ouverts, reprogrammés, modifiés, améliorés par les uns et les autres » $(2011: 117)$.

3. Cette notion - qu'il faut ici entendre dans son acception la plus large - est aujourd'hui fortement critiquée pour son ancrage mercatique et behavioriste : elle désigne spécifiquement l'utilisation de récompenses ou d'autres techniques de gratification inspirées du jeu vidéo pour favoriser un engagement total chez l'utilisateur. Pour décrire la multiplication et l'évolution des formes du ludique, Genvo (2011 et 2012) propose comme alternative le concept de " ludicisation », qui désigne l'ensemble des " processus qui consistent à faire entrer un objet dans le monde du jeu, en soulignant que la définition de cette notion et des objets auxquels elle renvoie est hautement contextuelle et peut être amenée à évoluer» (2011: 69). Pour une description critique de divers phénomènes de gamification, voir également Triclot (2011:230-236) 4. «Lorsqu'il actualise un jeu vidéo, le joueur va prendre connaissance du fonctionnement du système, de ses mécanismes (game) et va en éprouver le potentiel de "jeu" (la jouabilité) en adoptant une attitude ludique (play), ce que traduit le terme de gameplay en regroupant ces deux aspects dans une même notion de forme nominale » (Genvo, $2006: 215)$.

5. Ceux-ci ont été sélectionnés en raison de leur haut degré d'organisation (ils disposent d'un nom, de sites de référence, d'organes critiques, de travaux de recherche décrivant leurs enjeux,... ) par opposition aux formes de détournement qu'il n'est pas encore possible de classer dans une catégorie identifiable, tel que le récent «Twitch plays Pokémon» sur la plateforme Twitch, expérience qui a permis aux internautes connectés de diriger en même temps le personnage du jeu Pokémon Rouge (1996) et, malgré tout, de réussir à finir le jeu ; URL : http://www.twitch.tv/ twitchplayspokemon (signalons que tous les sites Internet mentionnés dans cet article ont été consultés pour la dernière fois le 23/03/2014).

6. 3744842 en janvier 2011 selon la page «FFN Research », sur Fan Fiction Statistics. URL : http:// ffnresearch.blogspot.be/.

7. Le terme provient de la contraction des mots «machine» et «cinema " (auxquels certains ajoutent encore le mot « animation »). Il aurait été créé dans les années 1990 par Hugh Hancock, lui-même producteur de machinimas et cofondateur du site Machinima.com. Toutefois, l'étymologie du terme ne fait pas tout à fait l'unanimité : voir Georges et Auray (2012a : 6).

8. On fait généralement remonter ce type d'usage au jeu DOOM (1993), qui avait la particularité d'offrir aux joueurs l'opportunité d'enregistrer une partie et de la partager avec les autres utilisateurs du logiciel, mais la pratique s'est surtout popularisée avec le jeu Quake (1996) car celui-ci comportait davantage de possibilités de paramétrage (voir Lowood, 2006 pour un historique plus détaillé).

9. La liste complète des vidéastes (ou groupes de vidéastes) dont les œuvres ont été étudiées se trouve en bibliographie.

10. Cf. Georges et Auray (2012a et 2012b), Lowood (2006 et 2008), Newman (2008), Nitsche (2007). 
11. Elles ont été récompensées lors de festivals dédiés au machinima, leurs auteurs ont été sollicités pour de nombreuses interviews et, dans le cas de Red vs. Blue, ont développé un modèle économique leur permettant de vivre de leurs productions. En réponse au succès des machinimas réalisés sur base du jeu Halo, Microsoft a en outre ajouté une commande permettant aux joueurs de baisser leurs armes : cette action ne comporte aucun intérêt ludique mais facilite le travail des machinimakers, qui l'utilisent pour donner l'illusion que leurs personnages sont en train de parler. 12. 32667 à la date du 23 mars 2014.

13. Speed Demos Archive. URL: http://speeddemosarchive.com/ et TASVideos. URL: http:// tasvideos.org/.

14. Ces notions sont ici employées dans leur acception sémiotique (voir Greimas et Courtés, 1979 : 420).

15. Dans le domaine précis des fanfictions, ce type de modèle généré par la communauté se nomme un fanon: " [the] fanon, the noncanonical knowledge about a source text, is the sum of the community's shared interpretive acts " (Kaplan, $2006: 136)$. Notons qu'il existe une tension continue entre l'interprétation individuelle de chaque amateur et celle, collective, qui domine dans la communauté : la seconde exerce un poids certain sur la première, qui peut néanmoins échapper aux attentes du collectif (pour éventuellement être récupérée par la suite, etc.). L'individuel et le communautaire s'entre-déterminent dans un mouvement aussi constant qu'imprévisible : « in this way, interpretive communities and their collective readings are never static, but rather function as ever-shifting intertexts for current and future fan interpretation and authorship » (Stein et Busse, 2009 : 198).

16. First-Person Shooter, ou jeu de tir à la première personne.

17. Voir Coppa (2006), Lowood (2006), Cameron et Carroll (2011) ou encore Newman (2008: 89-148) pour diverses mobilisations de cette notion dans le domaine des détournements médiatiques.

18. Au sujet du speedrun par exemple, «[...] as we examine this playing practice in more depth we might argue that speedrunning represents the ultimate expression of gamers' mastery of the system and of playing with videogames » (Newman, $2008: 129)$.

19. Ces connaissances et savoir-faire concernent également les failles n'ayant pas été rectifiées par les développeurs, comme le montre le mod «Unofficial Skyrim Patch », qui vient corriger divers bugs résiduels du jeu The Elder Scrolls V: Skyrim («Unofficial Skyrim Patch», sur Skyrim Nexus mods and community. URL : http://www.nexusmods.com/skyrim/mods/19/?).

20. Sur la notion de fanon, voir la note 15 .

21. L'auteure de la fiction "Crushcrushcrush», par exemple, prévient les critiques par la précaution suivante : "je sais que cette histoire est bourrée de défauts (et d'incohérences dont j'ai honte, et d'OoC, et de plein de choses encore XD) [...]» («Crushcrushcrush, chapitre 43 », sur Fanfiction.net. URL : https://www.fanfiction.net/s/7107693/43/Crushcrushcrush). Voir également les différentes mentions de l'OOC dans le sujet «Les OC /Original Character ou Personnage Original/ » sur le forum « Parlons fanfiction » : « à mon avis, mieux vaut un OC [original character, personnage créé par le fan et non par l'auteur d'origine] que trop d'OOC ! ; « l'objectif : pouvoir montrer des faiblesses du personnage canon, le risque étant dans ce cas de tomber dans l'OOC... [...] tout est une question de dosage »; etc. (sur Fanfiction.net. URL : https://www.fanfiction.net/ topic/77279/35495773/1/Les-OC-Original-Character-ou-Personnage-Original).

22. Commentaires que les lecteurs peuvent laisser en ligne à la suite des textes.

23. Il s'agit de lecteurs auxquels les auteurs de fanfictions peuvent soumettre leur texte avant de le publier pour que celui-ci soit critiqué et corrigé. Notons que cette appellation renvoie directement à celle de bêta-test, ce qui constitue un autre indice du mimétisme structurel qui caractérise les détournements de jeux vidéo par rapport au système dont ils sont issus. En effet, le bêta-test constitue la seconde période d'essai d'un logiciel, durant laquelle celui-ci est mis à 
l'épreuve par un grand nombre d'utilisateurs qui signalent aux concepteurs les derniers bugs et problèmes rencontrés afin de les résoudre avant la mise en vente.

24. Sur Fanfiction.net, avant de publier une fiction, l'auteur amateur se voit, par exemple, dans l'obligation d'approuver le règlement $\mathrm{du}$ site, au sein duquel il trouvera notamment les recommandations suivantes : « 1 . Spell check all story and poetry. There is no excuse for not performing this duty. If you do not have a word processor that has the spell checking feature, use a search engine such as Google.com to find one. 2. Proofread all entries for grammar and other aspects of writing before submission. "Hot off the press" content is often riddled with errors. No one is perfect but it is the duty of the writer to perform to the best of his/her ability ".

25. Pour illustration, le tool-assisted speedrun détenant actuellement le record de vitesse sur le jeu Castlevania: Aria of Sorrow (avec pour condition de finir le jeu intégralement) a nécessité pas moins de 179603 re-records (« Submission \#3039 : Kriole's GBA Castlevania : Aria of Sorrow “100 \% souls" in $24: 56.1 »$, sur TASVideos. URL : http://tasvideos.org/3039S.html).

26. Voir, pour exemple, les différents sujets du forum "Parlons fanfiction », sur Fanfiction.net. URL : https://www.fanfiction.net/forum/Parlons-fanfiction/77279/.

27. "Nexus Wiki ", sur Nexus mods and community. URL: http://wiki.tesnexus.com/index.php/ Main_Page.

28. À l'image de ce TAS consacré à Sonic Advance 2, dans la description duquel l'auteur fournit des cartes annotées des différents niveaux du jeu où la trajectoire de son personnage est comparée à celle du détenteur du précédent record: «Submission \#3426: Mukki's GBA Sonic Advance 2 in $18: 01.78 »$, sur TASVideos. URL : http://tasvideos.org/3426S.html.

29. Voir la série de vidéo : "DigitalPh33r's Guide to Making Halo 3 Machinima ", sur YouTube. URL : https://www.youtube.com/watch ?v =Eu3qxeZmBrk.

30. «À part ça, vu que je m'emmerde grave au lycée, je lance des défis à Crimson Warai et inversement. Vous pouvez les lire dans le recueil "Défis".=) Allez voir les siens, aussi " («Puisque la folie te guette, chapitre $30 »$, sur Fanfiction.net. URL : https://www.fanfiction.net/ s/5807613/30/Puisque-la-folie-te-guette).

31. Telles que l'«AkuRoku Day», date anniversaire, selon les fans, du couple formé par les personnages Axel et Roxas du jeu Kingdom Hearts. Celle-ci tombe le 13 août et est célébrée par les auteurs à travers l'écriture de textes mettant le couple en scène.

32. «Les petites bêtes", sur YouTube. URL: https://www.youtube.com/watch?v=HkkTlnAWsQ\&list=UUVuCJG5RPwnVXoqbwGSBoag.

33. «Des vacances qui commencent bien!", sur YouTube. URL: https://www.youtube.com/ watch?v=FGfofG81ip8\&index=8\&list=UU_ANJ8SEtUE8Y33x2LCXNwA.

34. «Common Sense Cooking with Carl the Cook », sur Internet Archive. URL : https://archive.org/ details/common_sense_cooking.

35. Les réalisateurs des vidéos tendent le plus souvent à se conformer aux conventions du septième art, notamment en ce qui concerne le positionnement de la caméra (utiliser l'alternance champ/contrechamp pour les dialogues, par exemple). Les "bandes annonces » réalisées par les producteurs de la série Red vs. Blue sont, à ce titre, des exemples parlants (voir notamment «Reconstruction Trailer», sur Rooster Teeth. URL: http://roosterteeth.com/ archive/?id=277\&v=more\&s=6). Pour d'autres illustrations de cette «intériorisation du langage cinématographique », voir notamment Georges et Auray (2012b : 11, version numérique) : « une raison de la consécration des machinimas est le fait qu'ils récupèrent des codes du langage cinématographique ». Voir également Di Crosta (2009: 27) : « en tant que produit d'une sousculture informatique, d'un savoir-faire technique appliqué aux techniques narratives cinématographiques, le machinima se situe à mi-chemin entre film et jeu vidéo ».

36. "This Spartan Life", sur Chaos Control Digizine. URL : http://www.chaoscontrol.com/thisspartan-life/. 
37. Dans Moltenbrey Karen (2005), "Out of Character ", Computer Graphics World, vol. 28, $\mathrm{n}^{\circ} 11$ [Disponible en ligne sur Computer Graphics World. URL: http://www.cgw.com/Publications/ CGW/2005/Volume-28-Issue-11-November-2005-/Out-of-Character.aspx].

38. « Guidelines ", sur TASVideos. URL : http://tasvideos.org/Guidelines.html.

39. «Frequently Asked Questions», sur Speed Demos Archive. URL: http:// www.speeddemosarchive.com/lang/faq_en.html.

40. On parle de scrolling forcé lorsque le décor d'un jeu se déplace indépendamment des mouvements de l'avatar, qui doit alors suivre le rythme de cette avancée sous peine d'échec

41. La vidéo suivante constitue une illustration parlante de cet usage : « NES Gradius in $10: 52.35$ by adelikat ", sur TASVideos. URL : http://tasvideos.org/Game/nes-gradius.html.

42. «SkyUI", sur Skyrim Nexus mods and community. URL : http://www.nexusmods.com/skyrim/ $\operatorname{mods} / 3863 /$ ?

43. "Alternate Start - Live Another Life", sur Skyrim Nexus mods and community. URL : http:// www.nexusmods.com/skyrim/mods/9557/?.

44. «Falskaar ", sur Skyrim Nexus - mods and community. URL : http://www.nexusmods.com/ skyrim/mods/37994/?

45. Ibid.

46. Dans ce deuxième cas, la parenté de la lecture et de la performance constitue une évidence puisque la consommation du mod consiste explicitement à jouer.

47. «Submission \#3557: Aglar's Genesis Sonic the Hedgehog 2 in 17 :51.6 », sur TASVideos. URL : http://tasvideos.org/3557S.html.

48. «TAS HD : Genesis Sonic the Hedgehog 2 in $17: 51.6$ by Aglar (2 screens) », sur YouTube. URL : https://www.youtube.com/watch ?v =cPN9fv8CAgw.

49. Pour preuve, ces quelques exemples issus des notes d'auteurs qui apparaissent en début ou en fin de chapitres, au sein même des fanfictions : «je suis surprise de voir que ma fic plait à tant de monde. Un grand merci à tous ceux qui me lisent et prennent la peine de me laisser une review. Ca me fait énormément plaisir à chaque fois. Merci aussi à ceux qui mettent mon histoire en favoris ou story/alert» («Kokoro ga nai, chapitre 6 », sur Fanfiction.net. URL : https:// www.fanfiction.net/s/5874607/6/Kokoro-ga-nai) ; « merci à mes premières lectrices, vous m'avez fait trop trop trop plaisiiiiiir ! (« Puisque la folie te guette, chapitre 2 », sur Fanfiction.net. URL : https://www.fanfiction.net/s/5807613/2/Puisque-la-folie-te-guette); «merci pour vos reviews adorables (auteur sautille) : Kaedegirl, Bouddha, Midna-Sama, LawSama, Nayru25, Ghostly Doll, Serya-chan et DuncanHeart [pseudonymes des commentateurs]» («Les Bannis ont droit d'amour, chapitre $3 »$, sur Fanfiction.net. URL: https://www.fanfiction.net/s/4975923/3/LesBannis-ont-droit-d-amour).

50. En voici quelques illustrations: «Je suis une anti-lecteurs fantômes, je trouve que c'est un profond manque de respect [...]»; «je souhaite m'inscrire, car je suis contre les lecteurs "fantômes" »; «je trouve que c'est une excellente idée d'avoir créé un groupe comme ça, au vu du nombre de lecteurs fantômes qui trainent sur ce site. [...] C'est personnel, mais je trouve que ne pas laisser de review à une fic qu'on a lu, c'est un peu comme ignorer quelqu'un qui est en train de nous parler». Réponses au sujet «Engagez-vous!» (pp.19-20), posté sur le forum «FIC », sur Fanfiction.net. URL : https://www.fanfiction.net/topic/77278/28145140/19/Engagezvous.

51. « FIC », sur Fanfiction.net. URL : https://www.fanfiction.net/forum/FIC/77278/.

52. «APAGCPMDEO », sur Fanfiction.net. URL : https://www.fanfiction.net/forum/ APAGCPMDEO/56737/.

53. "Requests for Constructive Criticism and Tips for Writing/Getting Reviews", sur Fanfiction.net. URL : https://www.fanfiction.net/topic/2872/8487857/1/Requests-forConstructive-Criticism-and-Tips-for-Writing-Getting-Reviews. 
54. Puisque le jeu n'existe pas en dehors de la partie, il est impératif, pour une œuvre vidéoludique, d'« [...] être reconnue comme jouable et [d']inciter le joueur à adopter une attitude ludique » (Genvo, 2008a : 5, version numérique).

55. Parmi les trente fictions du corpus, un tiers des textes comportent plus de cent mille mots et plus de la moitié dépassent les cinquante mille.

56. Voir, pour exemple, les différents chapitres de la fiction « Valse de la Lune », sur Fanfiction.net . URL : https://www.fanfiction.net/s/3690783/1/Valse-de-la-Lune.

57. C'est le cas dans plusieurs fictions de l'auteure Ariani Lee (voir son profil sur Fanfiction.net. URL : https://www.fanfiction.net/u/1331270/Ariani-Lee), ou encore dans « Les Bannis ont droit d'amour, chapitre $29 »$ (sur Fanfiction.net. URL : https://www.fanfiction.net/s/4975923/29/LesBannis-ont-droit-d-amour) ainsi que dans «Les Chroniques de l'Héritier Déchu» (sur Fanfiction.net. URL: https://www.fanfiction.net/s/4013327/1/Les-Chroniques-de-1-H \%C3 \% A9ritier-D \%C3 \%A9chu).

58. "Axel et Roxas dans cent thèmes, chapitre 2 ", sur Fanfiction.net. URL: https:// www.fanfiction.net/s/5360298/2/Axel-et-Roxas-dans-cent-th \%C3 \%A8mes.

59. «MAUVE, première partie : À cœur fendre, chapitre 1 ", sur Fanfiction.net. URL : https:// www.fanfiction.net/s/6422643/1/MAUVE-premi \%C3 \%A8re-partie-A-coeur-fendre.

60. «Axel et Roxas dans cent thèmes, chapitre 1 ", sur Fanfiction.net. URL: https:// www.fanfiction.net/s/5360298/1/Axel-et-Roxas-dans-cent-th \%C3 \%A8mes.

61. "I've lost my heart in winter, chapitre 1 », sur Fanfiction.net. URL: https:// www.fanfiction.net/s/5609560/1/I-ve-lost-my-heart-in-winter.

62. «Crushcrushcrush, chapitre $15 »$, sur Fanfiction.net. URL: https://www.fanfiction.net/ s/7107693/15/Crushcrushcrush.

63. «My Sweet Dangerous Cat, chapitre 1 », sur Fanfiction.net. URL : https://www.fanfiction.net/ s/4751757/1/My-Sweet-Dangerous-Cat.

64. C'est-à-dire ceux que Picard (2006), résumant la pensée de Cannon, oppose aux « machinimas artistiques »: ces derniers se caractériseraient par un plus haut degré d'abstraction et une primauté accordée à la recherche esthétique.

65. Voir, pour illustration, le premier épisode de la série : «Arby and The Chief : Season 1 », sur YouTube. URL: https://www.youtube.com/watch? $\mathrm{v}=\mathrm{kb} 8 \mathrm{rdc}-00 \mathrm{pQ}$ \&index=1\&list=PL104A38C965EDA0FC.

66. C'est notamment le cas dans les présentations des vidéos réalisées par le Numérimax Studio : « comme d'habitude j'attends impatiemment vos commentaires, idées, critiques, points positifs et négatifs, toujours dans l'optique de me remettre en cause et de progresser!» («[Machinima ${ }^{\mathrm{HD}}$ Halo 4] Poursuit», sur YouTube. URL: https://www.youtube.com/watch? $\mathrm{v}=\mathrm{E} 6 \mathrm{dJcV}$ wQ2Ik\&index $=18$ \&list $=$ UUfMaEkC5rPoX696mpJDI7gg) ; "j'espère donc que cela vous plaira! Comme d'habitude j'attends vos critique et remarque plus bas!» (« [Machinima] Le Mur (Spécial 700 Abonnés)», sur YouTube. URL : https://www.youtube.com/watch? $\mathrm{v}=$ NyH4IQal6a8\&list $=$ UUfMaEkC5rPoX696mpJDI7gg\&index $=4$ ); etc.

67. Michael Burns, créateur de la série Red vs. Blue, cité par Fitzgerald Courtney (2004), «Almost famous: Red vs. Blue's machinima stars", sur The Austin Chronicle. URL: http:// www.austinchronicle.com/screens/2004-03-05/200235/.

68. Générique de fin du machinima «Arby ' $n$ ' the Chief, Conflict - Part 1 » (08:28), sur YouTube. URL : https://www.youtube.com/watch?v=GsoXlpnPF90\&index=11\&list=PL104A38C965EDA0FC.

69. «RaceMenu ", sur Skyrim Nexus mods and community. URL: http://www.nexusmods.com/ skyrim/mods/29624/?.

70. «Frostfall - Hypothermia Camping Survival», sur Skyrim Nexus mods and community. URL : http://www.nexusmods.com/skyrim/mods/11163/?.

71. Celles-ci diffèrent pour chaque jeu. La description du speedrun du jeu Spelunker, entre autres, détaille les diverses stratégies adoptées par son auteur : «the central speedrunning strategy for 
this game revolves around manipulating the game's respawn system. Whenever you die, you will respawn from the location of the last item collected, excluding keys. [...] This allows a strategy of jumping over specific items (which requires precise timing) on the path to a key, then suiciding to avoid backtracking to the original path » («Spelunker », sur Speed Demos Archive. URL : http:// speeddemosarchive.com/Spelunker.html).

72. «Dragon Warrior", sur Speed Demos Archive. URL: http://speeddemosarchive.com/ DragonWarrior.html.

73. «Super Mario Bros», sur Speed Demos Archive. URL: http://speeddemosarchive.com/ Mario1.html.

74. «Welcome To TAS Videos", sur TASVideos. URL: http://tasvideos.org/ WelcomeToTASVideos.html.

75. Pour illustration, voir "SDA Knowledge Base», sur Speed Demos Archive. URL: https:// kb.speeddemosarchive.com/Main_Page.

76. « Debate : allowed or not?», sur TASVideos. URL : http://tasvideos.org/forum/viewtopic.php? $\mathrm{t}=14051$ \&postdays $=0$ \& postorder $=$ asc\&start $=0$ \&sid=2e04076f5242473eaf0abc8aac8b61a6.

77. «Interview of adelikat for GamersGlobal», sur TASVideos. URL: http://tasvideos.org/ Interviews/Adelikat/GamersGlobal2010.html.

78. «Submission \#2990: Ryuto's DS Brain Age in $06: 33.66 »$, sur TASVideos. URL: http:// tasvideos.org/2990S.html.

79. « About », sur This Spartan Life. URL : http://www.thisspartanlife.com/sample-page/.

80. C'est aussi le cas dans leurs interviews : " "I'm a failed independent filmmaker", says founder Burnie Burns, pointing to co-creator Jason Saldaña, “and he's a craft service guy whose claim to fame is listening to Britney Spears pee while singing 'Like a Virgin'." A third co-conspirator, Geoff Fink, is a tattooed ex-Army reporter, who affectionately christens Burns "a no-talent hack" ». Voir Fitzgerald Courtney (2004), « Almost famous : Red vs. Blue's machinima stars », sur The Austin Chronicle. URL : http://www.austinchronicle.com/screens/2004-03-05/200235/ (notons que l'interview en question est renseignée sur le site du studio : «Coverage », sur Rooster Teeth. URL : http://roosterteeth.com/about/coverage.php).

81. « Company History », sur Rooster Teeth. URL : http://roosterteeth.com/about/history.php.

82. Voir «Zardoz Partie 1 - Le film d'animation », sur Kingrabbit.fr. URL : http://kingrabbit.overblog.com/article-zardoz-partie-1-le-film-d-animation-93202605.html.

83. "TAS movies are art. Good TAS movies demonstrate TASing as art well. [...] TAS movies should be impressive, superhuman, unexpected » ("Interview of adelikat for GamersGlobal », sur TASVideos. URL : http://tasvideos.org/Interviews/adelikat/GamersGlobal2010.html).

84. «[...] in the past few years, a community of artists has created speedruns that go beyond what mere mortals could do». Teti John, «As Fast as Impossible : 10 Insanely Thrilling Tool-Assisted Speedruns », sur Crispy Gamer. URL : http://www.crispygamer.com/features/2009-04-20/as-fastas-impossible-10-insanely-thrilling-tool-assisted-speedruns.aspx.

85. «Are Tool-Assisted Runs Conceptual Art?», sur TASVideos. URL: http://tasvideos.org/ forum/viewtopic.php?t=14435.

86. Voir Delaney Kevin J. (9 avril 2004), «When Art Imitates Videogames, You Have "Red vs. Blue" », The Wall Street Journal, Dow Jones \& Company. [Disponible en ligne sur The Wall Street Journal. URL : http://online.wsj.com/articles/SB108145721789778243].

87. «Cooking Mama, The Unauthorized PETA Edition : Mama Kills Animals », sur PETA - People for the Ethical Treatment of Animals. URL : http://features.peta.org/CookingMama/.

88. Les jeux vidéo sont ici référencés selon le schéma suivant: Titre du jeu (développeur, éditeur [si celui-ci diffère du développeur], date de la première sortie). 


\section{RÉSUMÉS}

Cet article a pour objectif d'analyser conjointement quatre pratiques de détournement du jeu vidéo par les joueurs (la rédaction de fanfictions, la réalisation de machinimas, le modding, et le speedrun) en les envisageant comme des prolongements de l'expérience ludique. La forme des œuvres dérivées ainsi que les discours des joueurs qui les produisent seront étudiés parallèlement dans le but de mettre au jour en quoi ces pratiques renouvellent la conception même du jeu et lui ouvrent de nouveaux espaces. Ce travail se veut donc dans la lignée des play studies définies par Triclot (2011), qui envisagent le jeu vidéo non comme une forme figée mais comme une expérience.

This article aims at analysing four détournement practices in video games (fanfiction writing, machinima direction, modding, and speedrun), considering them as extensions of players' ludic experiences. Both the form of these derivative works and their players' discourses will be studied in parallel, in order to bring to light to what extent these practices are renewing the very notion of "game" and opening up new spaces for it. This work is therefore meant to be in line with play studies - as defined by Triclot (2011) -, which view video games not as static forms but as playful experiences.

\section{INDEX}

Mots-clés : détournement, play studies, machinimas, fanfictions, speedrun, modding

Keywords : détournement, play studies, machinimas, fanfictions, speedrun, modding

\section{AUTEUR}

\section{FANNY BARNABÉ}

Université de Liège, LEMME 